Check for updates

Cite this: RSC Adv., 2018, 8, 8721

Received 20th December 2017 Accepted 20th February 2018

DOI: 10.1039/c7ra13487h

rsc.li/rsc-advances

\title{
Eco-friendly reactions in PEG-400: a highly efficient and green approach for stereoselective access to multisubstituted 3,4-dihydro-2(1H)- quinazolines using 2-aminobenzylamines $\uparrow$
}

\begin{abstract}
Nutan Sharma, Pankaj Sharma and Sunita Bhagat (iD *
An efficient and stereoselective synthesis of novel 3,4-dihydro-2(1H)-quinazolines has been developed through cyclization reactions of 2 -aminobenzylamines with $\alpha$-oxoketene dithioacetals using PEG-400 as an inexpensive, easy to handle, non-toxic and recyclable reaction medium. The developed protocol is operationally simple and tolerates various substrates having different functionalities. This protocol features several attributes such as excellent yields, no work up, green reaction conditions, and being environmentally benign. The attractive feature of this new strategy is that all the reported final compounds have been isolated as single $(E)$-stereoisomeric forms, which was confirmed by ${ }^{1} \mathrm{HNMR}$ and $\mathrm{X}$-ray crystallographic studies.
\end{abstract}

\section{Introduction}

Quinazolines are a class of fused heterocyclic ring systems containing multiple pharmacophores which are undoubtedly well established as biologically and pharmaceutically important compounds. ${ }^{1}$ Quinazoline-based heterocycles and their hydrated congeners have been reported to possess diverse biological and therapeutic properties such as inhibition of the epidermal growth factor (EGF) receptors of tyrosine kinase, ${ }^{2 a}$ and analgesic, ${ }^{2 \boldsymbol{b}-\boldsymbol{d}}$ anticancer, ${ }^{3}$ anti-inflammatory, ${ }^{4}$ antidiuretic, ${ }^{5}$ anticonvulsant ${ }^{6}$ and anti-Alzheimer ${ }^{7}$ activities. Commercialized drug compounds such as gefitinib (I), erlotinib (II), alfuzosin (III), and afloqualone (IV) are some notable examples of drugs containing quinazoline as a core nucleus (Fig. 1). Considering these applications, development of novel synthetic routes towards the formation of these pharmaceutically important scaffolds attracts the interest of synthetic organic chemists.

Substituted quinazolines have been reported to be constructed by numerous methods involving several substrates. ${ }^{8-10}$ Among them, many of the synthetic methodologies have craftily employed 2-aminobenzylamines as substrates with aldehydes for the synthesis of quinazolines (Scheme $1, \mathrm{i}-\mathrm{iv}$ ). These reactions have been carried out by using (i) $\left[\mathrm{Cp}^{*} \mathrm{IrCl}_{2}\right]_{2}$ as a catalyst, four equivalents of styrene in xylene solvent under inert atmosphere of $\mathrm{N}_{2}, 24 \mathrm{~h}$, reflux conditions ${ }^{11 a}$ (Scheme 1, a); (ii)

Organic Synthesis Research Laboratory, Department of Chemistry, A. R. S. D. College, University of Delhi, New Delhi-110021, India. E-mail: sunitabhagat28@gmail.com $\dagger$ Electronic supplementary information (ESI) available: Datas and spectral copies of ${ }^{1} \mathrm{H},{ }^{13} \mathrm{C}$ NMR for target compounds. X-ray crystallographic data of compound 40 with CCDC No. 1055453. For ESI and crystallographic data in CIF or other electronic format see DOI: $10.1039 / \mathrm{c} 7 \mathrm{ra} 13487 \mathrm{~h}$ nanoclusters of bi-metallic Pt/Ir alloy as a catalyst, 5,5',6,6'-tetrahydroxy-3,3,3',3'-tetramethyl-1,1'-spiro-bisindane, (TTSBI) as a co-catalyst, $\mathrm{K}_{2} \mathrm{CO}_{3}$ as a base with $\mathrm{CDCl}_{3} / \mathrm{H}_{2} \mathrm{O}$ as solvent for a longer reaction duration ${ }^{11 b}$ (Scheme 1, b); (iii) a CuCl/DABCO/ 4-HO-TEMPO/O $\mathrm{O}_{2}$ catalytic system in $\mathrm{CH}_{3} \mathrm{CN}$ at $80{ }^{\circ} \mathrm{C}$ (ref. 12a) (Scheme 1, c) and (iv) four equivalents of $\mathrm{NaOCl}$ as oxidant in $\mathrm{MeOH}$ solvent for longer reaction hours ${ }^{12 b}$ (Scheme 1, d). However, despite the synthetic utility of these protocols, the reported techniques suffer from drawbacks such as the use of expensive metal catalyst along with a co catalyst and requirement of a stoichiometric amount of toxic oxidant $\mathrm{NaOCl}$ for the transformation to take place. In such a scenario, development of a catalyst free and eco friendly methodology to deliver functionalized quinazolines will provide an intriguing and green alternative to the conventional approach.

Over the years, there has been a growing public concern towards the adverse effects of toxic and volatile organic solvents<smiles>COc1cc2ncnc(Nc3ccc(F)c(C)c3)c2cc1OCCCN1CCOCC1</smiles>

(I) Gefitinib<smiles>C#Cc1cccc(Nc2c(OCCOC)c(OCCOC)cc3cncnc23)c1</smiles>

(II) Erlotinib

(IV) Afloqualone

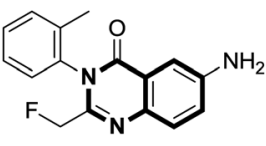

Fig. 1 Some popular marketed drugs containing quinazoline as a core nucleus. 
i. Previous synthetic approaches

(a)

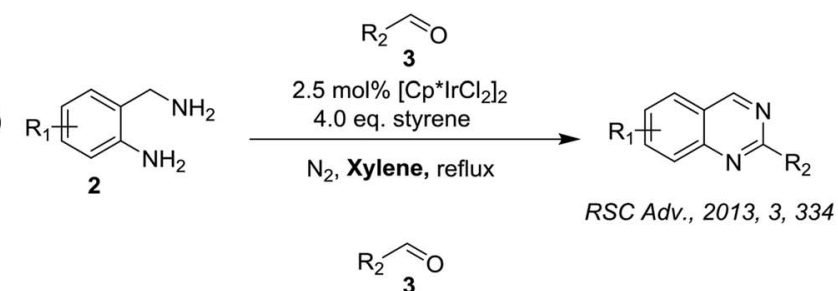

(b)
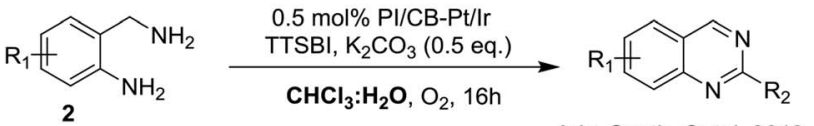

Adv. Synth. Catal. 2012, 354, 2899

(c)

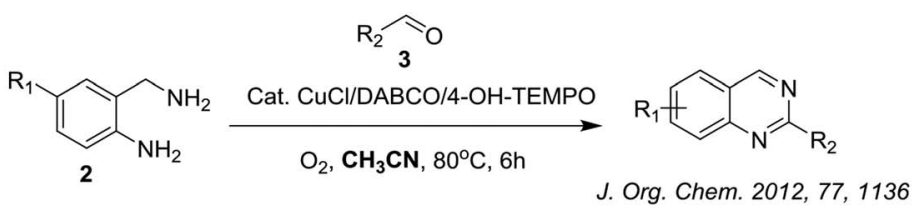

(d)
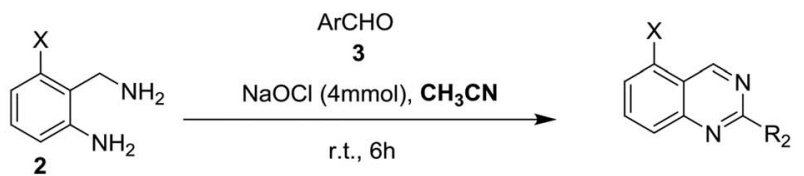

Adv. Synth. Catal. 2010, 352, 341

This Work :

(e)

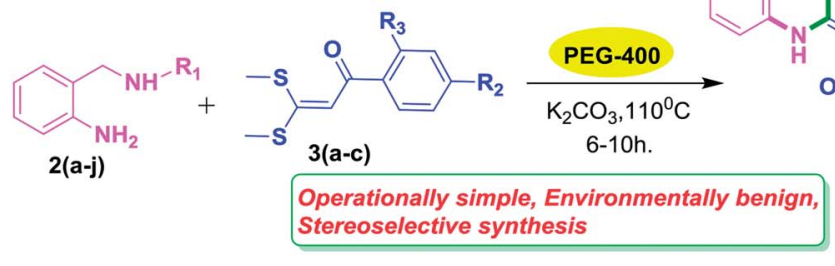

$R_{1}=H$, methyl, ethyl, n-propyl, isopropyl, n-butyl, tert.-butyl, cyclopropyl, cyclohexyl, benzyl

$\mathbf{R}_{\mathbf{2}}=\mathbf{F}, \mathrm{CF}_{3}$

$\mathbf{R}_{3}=\mathrm{OCH}_{3}$

Scheme 1 Previous synthetic approaches and our designed approach.

and the search for alternate nonhazardous reaction media is gaining progress. Meanwhile, using water as a green solvent has been well documented ${ }^{13}$ but its practical use is limited due to the hydrophobic nature of organic compounds. ${ }^{14}$ On the contrary, PEGs have been explored as powerful, novel, ecofriendly reaction media for innumerable organic transformations ${ }^{15}$ owing to their non toxic, inexpensive, thermally stable, readily recyclable, and biodegradable nature. ${ }^{16}$ In continuation of our research studies aimed at developing environmentally benign and improved synthetic methodologies for organic reactions, ${ }^{17}$ we herein report for the first time an efficient and green approach for the stereoselective synthesis of 3,4-dihydro-2 $(1 H)$-quinazolines via substituted 2 -amino benzylamines and $\alpha$-oxoketene dithioacetals as building blocks using PEG-400 as an eco-friendly reaction medium (Scheme 1, e). To the best of our knowledge, there have been no reports on the synthesis of 3,4-dihydro-2(1H)-quinazolines using PEG-400 as a solvent under catalyst-free conditions.

\section{Results and discussion}

To check feasibility of this route, a model reaction between $\mathbf{2 b}$ and 3a was performed by varying different protic and aprotic solvents and various bases (Table 1). When the reaction was performed using EtOH as polar protic solvent and $\mathrm{CH}_{3} \mathrm{CN}$ as low boiling aprotic solvent under reflux conditions using $\mathrm{K}_{2} \mathrm{CO}_{3}$ as base, the desired product $\mathbf{4 b}$ was obtained in lower yields (Table 1, entries 1 and 2). Whereas under similar conditions, high boiling aprotic solvent such as toluene was not proven very effective and gave the desired product $4 \mathrm{~b}$ in $50 \%$ yield after $12 \mathrm{~h}$ (Table 1, entry 3). High boiling polar aprotic solvents like DMF and DMSO afforded the desired product in higher yield taking less time (Table 1, entries 4 and 5). It should be noted that use of polar protic solvents like $n$ - $\mathrm{BuOH}$ and $t-\mathrm{BuOH}$ increased the yield to $65 \%$ and $70 \%$ in the presence of $\mathrm{K}_{2} \mathrm{CO}_{3}$ as base in $10 \mathrm{~h}$ (Table 1, entries 6 and 7). Keeping in view the economical and environmental concerns, $\mathrm{H}_{2} \mathrm{O}$ was screened as the reaction 
Table 1 Optimization of reaction conditions ${ }^{a}$

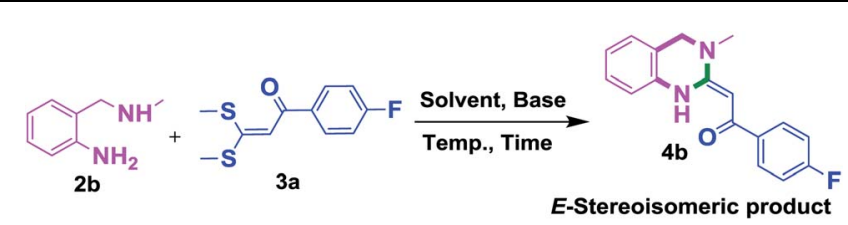

\begin{tabular}{llllll}
\hline Entry & Solvent & Base & Temp $\left({ }^{\circ} \mathrm{C}\right)$ & Time $(\mathrm{h})$ & Yield $^{b}(\%)$ \\
\hline 1 & EtOH & $\mathrm{K}_{2} \mathrm{CO}_{3}$ & 80 & 15 & 40 \\
2 & $\mathrm{CH}_{3} \mathrm{CN}$ & $\mathrm{K}_{2} \mathrm{CO}_{3}$ & 90 & 18 & 45 \\
3 & Toluene & $\mathrm{K}_{2} \mathrm{CO}_{3}$ & 110 & 12 & 50 \\
4 & DMF & $\mathrm{K}_{2} \mathrm{CO}_{3}$ & 120 & 10 & 60 \\
5 & DMSO & $\mathrm{K}_{2} \mathrm{CO}_{3}$ & 120 & 10 & 62 \\
6 & $n$-BuOH & $\mathrm{K}_{2} \mathrm{CO}_{3}$ & 120 & 10 & 65 \\
7 & $t$-BuOH & $\mathrm{K}_{2} \mathrm{CO}_{3}$ & 85 & 10 & 70 \\
8 & $\mathrm{H}$ O & $\mathrm{K}_{2} \mathrm{CO}_{3}$ & 100 & 15 & Trace \\
9 & PEG 400 & $\mathrm{K}_{2} \mathrm{CO}_{3}$ & $\mathbf{1 1 0}$ & $\mathbf{8}$ & $\mathbf{9 0}$ \\
10 & PEG 400: $\mathrm{H}_{2} \mathrm{O}$ & $\mathrm{K}_{2} \mathrm{CO}_{3}$ & 110 & 8 & 81 \\
11 & PEG 400: EtOH & $\mathrm{K}_{2} \mathrm{CO}_{3}$ & 110 & 10 & 72 \\
12 & PEG 400 & $\mathrm{K}_{2} \mathrm{CO}_{3}$ & 120 & 8 & 90 \\
13 & PEG 400 & $\mathrm{K}_{2} \mathrm{CO}_{3}$ & 90 & 8 & 80 \\
14 & PEG 400 & $\mathrm{Na}_{2} \mathrm{CO}_{3}$ & 110 & 8 & 85 \\
15 & PEG 400 & $\mathrm{TEA}^{2}$ & 110 & 8 & 65 \\
16 & PEG 400 & $\mathrm{DIPEA}^{2}$ & 110 & 8 & 68 \\
17 & PEG 400 & - & 110 & 8 & 20
\end{tabular}

${ }^{a}$ Reactions were performed using 1 equiv. of $3 \mathbf{a}, 1.2$ equiv. of $2 \mathbf{b}, 3.0$ equiv. of base. ${ }^{b}$ Isolated yield.

medium but observations indicated trace amount of product formation due to solubility problem (Table 1 , entry 8 ). In order to improve the efficiency of the reaction, we next performed our model reaction in PEG-400 at $110{ }^{\circ} \mathrm{C}$ using $\mathrm{K}_{2} \mathrm{CO}_{3}$ as base, and to our delight corresponding product $\mathbf{4 b}$ was obtained in a maximum yield of $90 \%$ within $8 \mathrm{~h}$ (Table 1, entry 9). Encouraged by the above result, a mixture of PEG-400 and $\mathrm{H}_{2} \mathrm{O}$ $(1: 1)$, was used as reaction medium which resulted in the formation of product $\mathbf{4 b}$ in $81 \%$ yield (Table 1, entry 10). Moreover, change of combination of solvents from PEG-400 and $\mathrm{H}_{2} \mathrm{O}$ to PEG-400 and EtOH $(1: 1)$ also had a detrimental effect on the yield of the product (Table 1, entry 11). Further increase or decrease in the temperature of the reaction could not increase the yield of the desired product $4 \mathbf{b}$ (Table 1, entries 12 and 13). From Table 1, it is clear that PEG-400 unanimously dominates as effective solvent medium because it increases the solubility of reactants, which leads to larger interfacial area and lower mass transfer resistance. ${ }^{18}$ When base was changed from $\mathrm{K}_{2} \mathrm{CO}_{3}$ to $\mathrm{Na}_{2} \mathrm{CO}_{3}$ at $110{ }^{\circ} \mathrm{C}, 85 \%$ yield was observed in $8 \mathrm{~h}$ (Table 1 , entry 14$)$.

Screening of other organic bases such as TEA and DIPEA in same reaction conditions resulted in $65 \%$ and $68 \%$ yields respectively (Table 1, entries 15 and 16). However the yield of reaction was drastically reduced to $20 \%$ when the reaction was performed in the absence of base (Table 1, entry 17). These optimization studies led to the conclusion that PEG-400 with $\mathrm{K}_{2} \mathrm{CO}_{3}$ is the reaction medium of choice for this transformation. The reaction product was fully characterized by the IR, ${ }^{1} \mathrm{H}$ NMR,
${ }^{13} \mathrm{C}$ NMR \& HRMS data. Compound $\mathbf{4 b}$ existed in one stereoisomeric form ( $E$-form) as evident from its IR \& ${ }^{1} \mathrm{HNMR}$ data. IR spectra strongly indicated a hydrogen-bonded carbonyl stretching vibration, which was merged with bands around and below $1600 \mathrm{~cm}^{-1}\left(1594 \mathrm{~cm}^{-1}\right.$ in this case). The signals for vinylic proton appeared as sharp singlets, indicating the purity of the geometrical isomers. ${ }^{1}$ HNMR spectrum showed a characteristic chelated $-\mathrm{NH}$ proton far downfield at $\delta 13.66$, assigned to the amino group which participated in a strong hydrogen bond with oxygen of the carbonyl group in a six membered, planar chelate ${ }^{19}$ (Fig. 2). Moreover, ${ }^{1}$ HNMR analysis of crude reaction mixture of $\mathbf{4 g}$ (see ESI, $\uparrow$ page 31) also indicated the formation of one stereoisomeric form of the product. Its spectral data analysis confirmed the structure.

In the ${ }^{1} \mathrm{H}$ NMR spectrum, presence of peaks as sharp singlets at $\delta 5.43(\mathrm{~s}, 1 \mathrm{H})$ and $\delta 4.45(\mathrm{~s}, 2 \mathrm{H})$, were characterized for vinylic proton and methylene group $-\mathrm{NCH}_{2}{ }^{-}$in $\mathbf{4 b}$. In the ${ }^{13} \mathrm{C} \mathrm{NMR}$ spectrum of $\mathbf{4 b}$, characteristic peak at $\delta 75.9$ and $\delta 50.7$ was observed for vinylic carbon and methylene carbon. HRMS data of the compound showed $\mathrm{M}+1$ peak. Similarly all other compounds were also characterized by IR, ${ }^{1} \mathrm{H}$ NMR, ${ }^{13} \mathrm{C}$ NMR and HRMS studies. The structure of the product $4 \mathbf{0}$ was determined by single crystal X-ray diffraction (Fig. 3). The structures of the other products were concluded by analogy.

With these optimized conditions in hand, the scope and robustness of the protocol was explored by employing diversely substituted 2-amino benzylamines $2 \mathbf{2 a}-\mathbf{j}$ with $\alpha$-oxoketene dithioacetals 3a-c (Table 2). We observed that amines 2a-g having aliphatic substitution such as methyl, ethyl, $n$-propyl, isopropyl, $n$-butyl and tert-butyl at $\mathbf{R}^{\mathbf{1}}$ afforded the desired stereoselective products $\mathbf{4 a - g}, \mathbf{4 l} \& \mathbf{4 n}$ in good to excellent yields of $80-92 \%$ with $\alpha$-oxoketene dithioacetals 3a-c (Table 2, entries $1-7,12,14)$. However, the same $\alpha$-oxoketene dithioacetals 3a-c provided the products $\mathbf{4 h}-\mathbf{k}, \mathbf{4 m} \& \mathbf{4 0}-\mathbf{p}$ in comparatively lower yields of $65-71 \%$ when amines $2 \mathbf{h}-\mathbf{j}$ bearing aromatic and cyclic

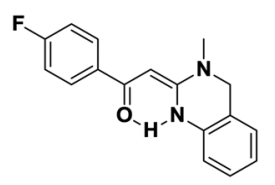

Fig. 2 Stereoselectivity via formation of six membered planar chelate.

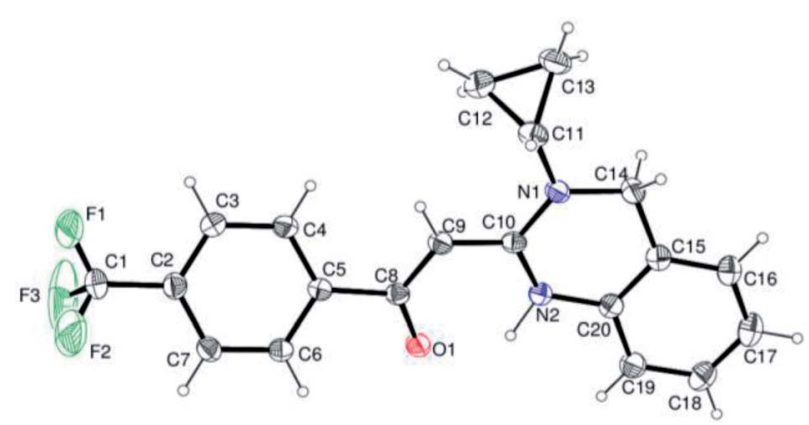

Fig. 3 Ortep diagram of compound 40. 
View Article Online

RSC Advances

Paper

Table 2 Substrate scope of substituted 3,4-dihydro-2(1H)-quinazolines ${ }^{a}$
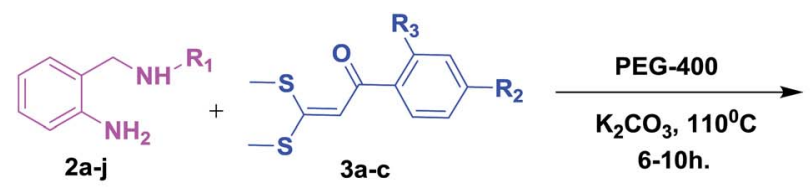<smiles>[R4]c1ccc(C(=O)/C=C2/Nc3ccccc3CN2[R])c([R3])c1</smiles>

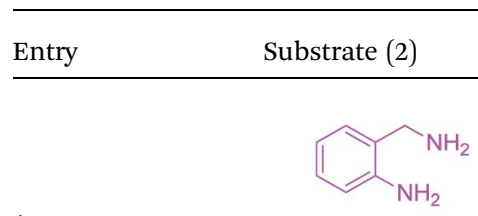

1

$2 a$

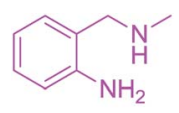

Lb

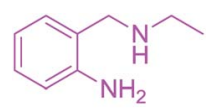

4

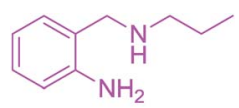

rd

$3 a$

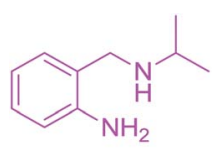

$3 a$

$2 \mathrm{e}$

6

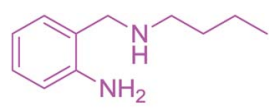

of

Ba

Ba

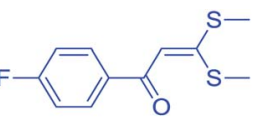

$3 a$

$3 \mathbf{a}$

Product (4)

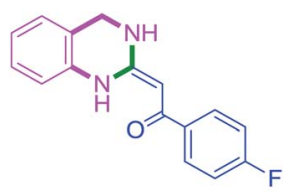

Yield $^{b}(\%)$

80

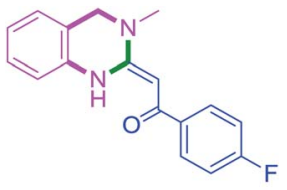

90

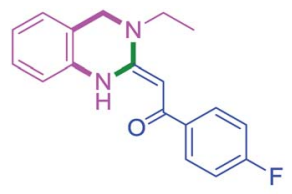

88

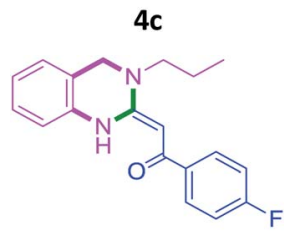

85

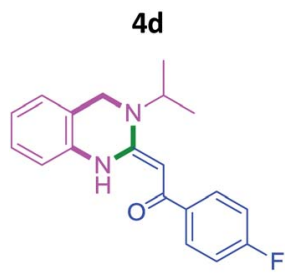

82

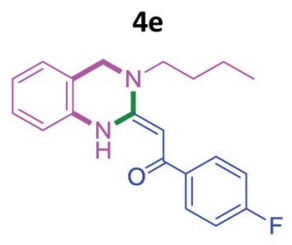

92

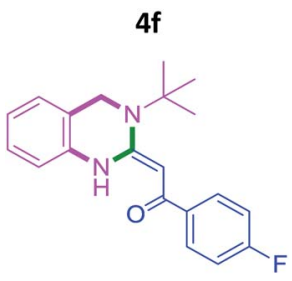

$4 \mathrm{~g}$

8724 | RSC Adv., 2018, 8, 8721-8731

This journal is @ The Royal Society of Chemistry 2018 
Paper

View Article Online

RSC Advances

Table 2 (Contd.)

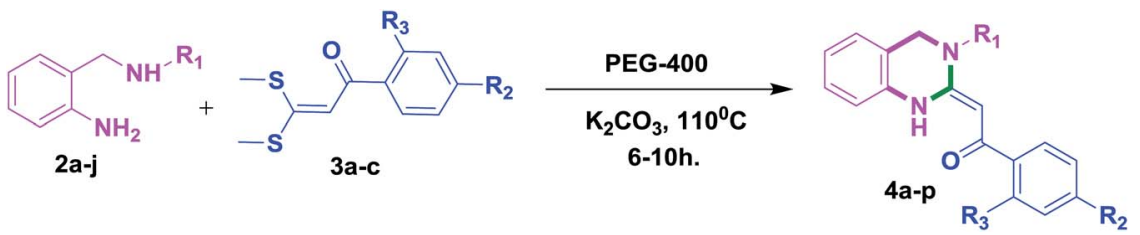

Entry Substrate (2)

$3 a$

$2 \mathrm{~h}$

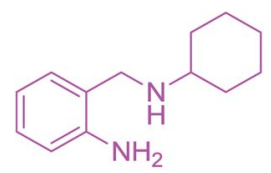

$2 \mathbf{i}$

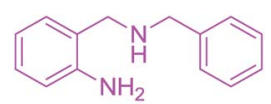

10
$3 a$

$3 a$

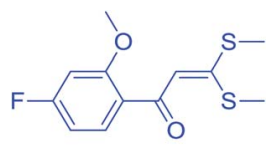

sb
12

if

13

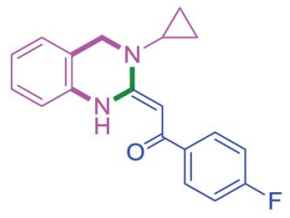

th
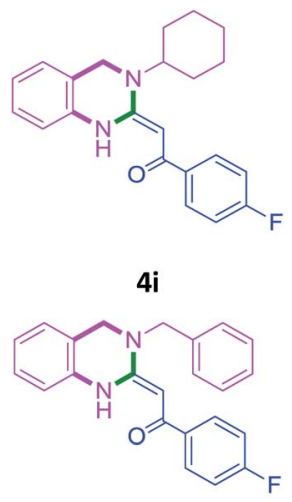

$4 j$
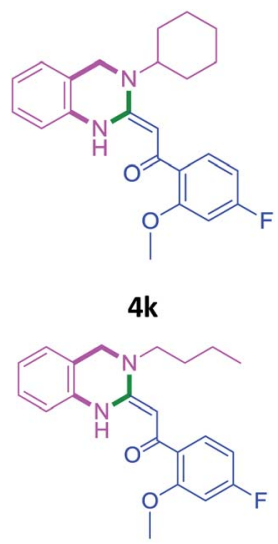

41

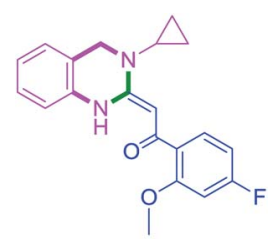

$4 m$
70

71

68

65

82

70

This journal is (c) The Royal Society of Chemistry 2018

RSC Adv., 2018, 8, 8721-8731 | 8725 


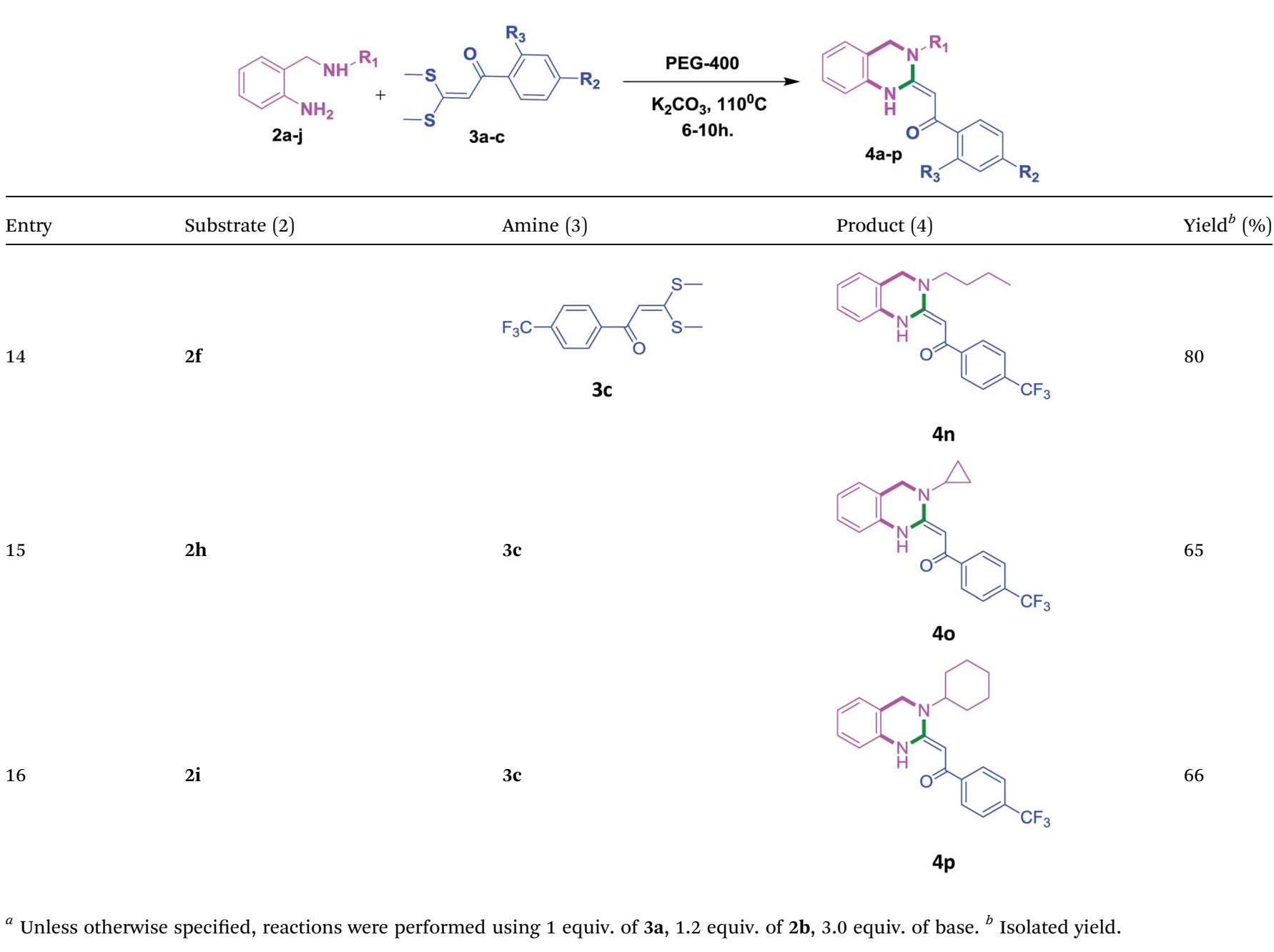

groups such as benzyl, cyclopropyl and cyclohexyl at $\mathbf{R}^{\mathbf{1}}$ were used (Table 2, entries $8-11,13,15-16$ ). This can be attributed to the lesser reactivity of aromatic and cyclic amines due to the involvement of lone pair of $-\mathrm{NH}_{2}$ in conjugation in the former and attachment of $-\mathrm{NH}_{2}$ group to a $\mathrm{sp}^{2}$ hybridized carbon atom in the later as compared to aliphatic amines.

In order to justify sustainable chemistry issues, recovery and reusability of PEG-400 was studied using the reaction of $2 \mathbf{g} \& \mathbf{3 a}$ in the presence of PEG-400 $(10 \mathrm{ml})$ at $110^{\circ} \mathrm{C}$ for $6-10 \mathrm{~h}$. After the completion of reaction, reaction mass was then cooled and solvent was evaporated under vacuum. Since PEG is immiscible with diethyl ether, it was washed (two times) with this solvent to remove any unwanted organic impurities and was used successfully in consecutive runs (the yield decreased from $86 \%$ to $72 \%$ after 5 runs, Table 3), although a weight loss of

Table 3 Recycling of PEG-400

\begin{tabular}{llllll}
\hline Runs & 1 & 2 & 3 & 4 & 5 \\
\hline Yield (\%) & 86 & 84 & 80 & 76 & 72
\end{tabular}

approximately 5\% of PEG was observed from cycle to cycle due to mechanical loss.

A plausible mechanism for the 3,4-dihydro-2 $(1 H)$-quinazoline ring formation is depicted in Scheme 2. The mechanism first involves the substitution of the thiomethyl group of $\alpha$ oxoketene dithioacetal 3 by that amino group of reactant 2 which is directly attached to aromatic ring, resulting in the formation of intermediate $\mathbf{4 A}$ at first.

This can be assigned to the presence of characteristic chelated proton of $-\mathrm{NH}$ at very high chemical shift values $(\delta 14-$

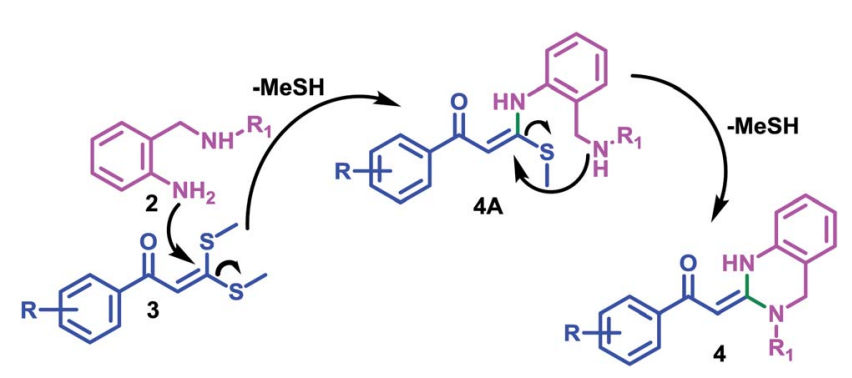

Scheme 2 Plausible Mechanism. 
$15 \mathrm{ppm})$ in the ${ }^{1} \mathrm{HNMR}$ data of compounds $\mathbf{4}(\mathbf{a}-\mathbf{p})$. After that, second thiomethyl group is replaced by second amino group of reactant 2 resulting in formation of cyclized product 4 with subsequent elimination of $-\mathrm{MeSH}$.

\section{Conclusions}

In conclusion, we have succeeded in developing first novel, inexpensine, cleaner and green methodology for the synthesis of 3,4dihydro- $2(1 H)$-quinazolines. Moreover, avoid of work-up make this new strategy attractive, easy to execute and facile for the assembly of a wide variety of biologically relevant dihydroquinazolines. As the methodology is completely devoid of anhydrous solvents or any catalysts, this approach therefore exemplifies the reconciliation of operational simplicity and economic viability in an environmental friendly time and cost effective manner. It is noteworthy that $\alpha$ oxoketene dithioacetals and substituted 2-amino benzylamines selectively afforded a single isomer. Further applicability of PEG400 as solvent system to a wide range of substrates towards the development of catalyst free pathways for synthesizing diverse range of biologically important heterocycles is an ongoing goal of research in our laboratory.

\section{Experimental}

\section{General information and method}

All the reactions were performed in an oven-dried Schlenk flask under nitrogen atmosphere. Column chromatography was performed using silica gel (mesh 100-200). TLC analysis was performed on commercially prepared 60 F254 silica gel plates. Visualization of spots on TLC plate was accomplished with UV light $(254 \mathrm{~nm})$ and staining over $\mathrm{I}_{2}$ chamber. IR spectra was recorded in $\mathrm{CHCl}_{3}$ on a Perkin Elmer Spectrum RX-1 FT-IR spectrophotometer. $\mathrm{CDCl}_{3}$ was used as NMR solvent for characterization of compounds. The ${ }^{1} \mathrm{H}$ NMR spectra were recorded on a Jeol JNM-ECX400P at $400 \mathrm{MHz}$. The ${ }^{13} \mathrm{C}$ NMR spectra on Jeol JNM-ECX400P at $100 \mathrm{MHz}$. Chemical shifts for carbons are reported in ppm from tetramethylsilane and are referenced to the carbon resonance of the solvent. Data are reported as follows: chemical shift, multiplicity ( $\mathrm{s}=$ singlet, $\mathrm{d}=$ doublet, $\mathrm{t}$ $=$ triplet, $\mathrm{q}=$ quartet, $\mathrm{m}=$ multiplet, $\mathrm{dd}=$ doublet of doublet, br $\mathrm{s}=$ broad singlet), coupling constants in Hertz, and integration. HRMS (ESI) were recorded with Q-TOF electrospray mass spectrometer. All purchased chemicals were used as received. All melting points are uncorrected.

\section{General procedure for synthesis of $2-\mathrm{N}$-aryl/alkyl substituted anilines $(2 \mathbf{a}-\mathbf{j})$}

Compounds $\mathbf{2 a}-\mathbf{j}$ were synthesized by reductive amination ${ }^{20}$ of 2-nitro benzaldehyde with various aliphatic, aromatic and cyclic amines followed by reduction ${ }^{21}$ of nitro group using $10 \% \mathrm{Pd}-\mathrm{C} /$ $\mathrm{H}_{2}$ in $\mathrm{EtOH}$ at 50 Psi for 2-3 h. Formation of compounds $2 \mathbf{2}-\mathbf{j}$ was confirmed by ${ }^{1} \mathrm{H}$ NMR, ${ }^{13} \mathrm{C}$ NMR and HRMS data.

\section{Analytical data of 2-amino benzylamines}

2-(aminomethyl)aniline (2a). The compound was obtained as a light brown solid, yield: $80 \% ;{ }^{1} \mathrm{H}$ NMR (400 MHz, DMSO-d ${ }_{6}$ ) $\delta: 7.03(\mathrm{dd}, J=7.33,1.37 \mathrm{~Hz}, 1 \mathrm{H}), 6.94(\mathrm{td}, J=7.79,1.37 \mathrm{~Hz}, 1 \mathrm{H})$, $6.60(\mathrm{dd}, J=7.79,0.92 \mathrm{~Hz}, 1 \mathrm{H}), 6.50(\mathrm{td}, J=7.33,0.92 \mathrm{~Hz}, 1 \mathrm{H})$, $4.98\left(\mathrm{t}, J=5.50 \mathrm{~Hz}, 2 \mathrm{H},-\mathrm{CH}_{2} \mathrm{NH}_{2}-\right), 4.88\left(\mathrm{bs}, 2 \mathrm{H},-\mathrm{NH}_{2}\right), 4.36(\mathrm{~s}$, $\left.2 \mathrm{H},-\mathrm{CH}_{2} \mathrm{NH}_{2}-\right) ;{ }^{13} \mathrm{C}$ NMR (100 MHz, DMSO-d $\left.{ }_{6}\right) \delta: 146.3,127.7$, 127.6, 125.3, 115.8, 114.5, 61.2 (benzylic- $\mathrm{CH}_{2}-$ ); HRMS (ESI) (M $+\mathrm{H})^{+}$calcd for $\mathrm{C}_{7} \mathrm{H}_{10} \mathrm{~N}_{2}$ : 123.0922, found 123.0913.

2-((methylamino)methyl)aniline (2b). The compound was obtained as a dark green solid, yield: $85 \% ;{ }^{1} \mathrm{H}$ NMR $(400 \mathrm{MHz}$, DMSO-d $\left._{6}\right) \delta: 7.20(\mathrm{dd}, J=7.33,1.37 \mathrm{~Hz}, 1 \mathrm{H}), 7.05(\mathrm{td}, J=7.33$, $1.37 \mathrm{~Hz}, 1 \mathrm{H}), 6.70(\mathrm{dd}, J=8.24,1.37 \mathrm{~Hz}, 1 \mathrm{H}), 6.54(\mathrm{td}, J=7.33$, $1.37 \mathrm{~Hz}, 1 \mathrm{H}), 3.96\left(\mathrm{~s}, 2 \mathrm{H}\right.$, benzylic- $\left.\mathrm{CH}_{2}-\right), 2.49$ (s, 3H, $-\mathrm{NHCH}_{3}$ ); ${ }^{13} \mathrm{C}$ NMR (100 MHz, DMSO-d ${ }_{6}$ ) $\delta: 147.7,132.0,129.8,116.1$, 115.8, 115.2, 47.5 (benzylic- $\left.\mathrm{CH}_{2}-\right)$, $32.0\left(-\mathrm{NHCH}_{3}\right)$; HRMS (ESI) $(\mathrm{M}+\mathrm{H})^{+}$calcd for $\mathrm{C}_{8} \mathrm{H}_{12} \mathrm{~N}_{2}:$ 137.1078, found 137.1073.

2-((ethylamino)methyl)aniline (2c). The compound was obtained as a dark green solid, yield: $83 \% ;{ }^{1} \mathrm{H}$ NMR $(400 \mathrm{MHz}$, DMSO-d $\left._{6}\right) \delta: 7.16(\mathrm{~d}, J=7.79 \mathrm{~Hz}, 1 \mathrm{H}), 7.02(\mathrm{t}, J=8.24 \mathrm{~Hz}, 1 \mathrm{H})$, $6.68(\mathrm{~d}, J=7.79 \mathrm{~Hz}, 1 \mathrm{H}), 6.55(\mathrm{t}, J=7.33 \mathrm{~Hz}, 1 \mathrm{H}), 5.69(\mathrm{bs}, 2 \mathrm{H}$, $-\mathrm{NH}_{2}$ ), $3.86\left(\mathrm{~s}, 2 \mathrm{H}\right.$, benzylic- $\left.\mathrm{CH}_{2}-\right), 2.81$ (q, $J=7.33 \mathrm{~Hz}, 2 \mathrm{H}$, $\left.-\mathrm{CH}_{2} \mathrm{CH}_{3}\right), 1.16\left(\mathrm{t}, J=7.33 \mathrm{~Hz}, 3 \mathrm{H},-\mathrm{CH}_{3}\right) ;{ }^{13} \mathrm{C}$ NMR $(100 \mathrm{MHz}$, $\mathrm{DMSO}_{6}$ ) $\delta$ : 147.6, 131.0, 129.5, 128.8, 117.4, 115.4, 47.2 (benzylic- $\left.\mathrm{CH}_{2}-\right), 42.1\left(-\mathrm{CH}_{2} \mathrm{CH}_{3}\right), 11.9\left(-\mathrm{CH}_{3}\right)$; HRMS (ESI) (M + $\mathrm{H})^{+}$calcd for $\mathrm{C}_{9} \mathrm{H}_{14} \mathrm{~N}_{2}$ : 151.1235, found 151.1228.

2-((propylamino)methyl)aniline (2d). The compound was obtained as a dark green solid, yield: $80 \% ;{ }^{1} \mathrm{H}$ NMR $(400 \mathrm{MHz}$, DMSO-d $\left._{6}\right) \delta: 7.21(\mathrm{~d}, J=7.79 \mathrm{~Hz}, 1 \mathrm{H}), 7.01(\mathrm{t}, J=8.24 \mathrm{~Hz}, 1 \mathrm{H})$, $6.68(\mathrm{~d}, J=8.24 \mathrm{~Hz}, 1 \mathrm{H}), 6.53(\mathrm{t}, J=7.33 \mathrm{~Hz}, 1 \mathrm{H}), 3.94(\mathrm{~s}, 2 \mathrm{H}$, benzylic- $\left.\mathrm{CH}_{2}-\right), 2.79\left(\mathrm{t}, 2 \mathrm{H}, J=7.79 \mathrm{~Hz},-\mathrm{CH}_{2} \mathrm{CH}_{2} \mathrm{CH}_{3}\right), 1.65$ (sext, $\left.2 \mathrm{H}, J=7.79 \mathrm{~Hz},-\mathrm{CH}_{2} \mathrm{CH}_{2} \mathrm{CH}_{3}\right), 0.84(\mathrm{t}, 3 \mathrm{H}, J=7.33 \mathrm{~Hz}$, $\left.-\mathrm{CH}_{3}\right) ;{ }^{13} \mathrm{C}$ NMR $\left(100 \mathrm{MHz}, \mathrm{DMSO}-\mathrm{d}_{6}\right) \delta: 147.4,132.7,131.8$, 119.5, 118.1, 116.9, $49.8\left(-\mathrm{CH}_{2} \mathrm{CH}_{2} \mathrm{CH}_{3}\right), 47.8$ (benzylic- $\mathrm{CH}_{2}-$ ), $20.1\left(-\mathrm{CH}_{2} \mathrm{CH}_{2} \mathrm{CH}_{3}\right), 11.9\left(-\mathrm{CH}_{3}\right)$; HRMS (ESI) $(\mathrm{M}+\mathrm{H})^{+}$calcd for $\mathrm{C}_{10} \mathrm{H}_{16} \mathrm{~N}_{2}$ : 165.1391, found 165.1379.

2-((isopropylamino)methyl)aniline (2e). The compound was obtained as dark green solid, yield: $81 \%$; ${ }^{1} \mathrm{H}$ NMR $(400 \mathrm{MHz}$, $\left.\mathrm{CDCl}_{3}\right) \delta: 7.16(\mathrm{~d}, J=7.79 \mathrm{~Hz}, 1 \mathrm{H}), 7.02(\mathrm{t}, J=8.24 \mathrm{~Hz}, 1 \mathrm{H}), 6.68$ $(\mathrm{d}, J=7.79 \mathrm{~Hz}, 1 \mathrm{H}), 6.55(\mathrm{t}, J=7.33 \mathrm{~Hz}, 1 \mathrm{H}), 3.92(\mathrm{~s}, 2 \mathrm{H}$, benzylic- $\left.\mathrm{CH}_{2}-\right)$, 4.29-4.23 (m, $\left.1 \mathrm{H},-\mathrm{NHCH}\right), 1.25(\mathrm{~s}, 6 \mathrm{H}, 2 \times$ $\mathrm{CH}_{3}$ ); ${ }^{13} \mathrm{C}$ NMR (100 MHz, DMSO-d $\left.{ }_{6}\right) \delta: 147.6,131.0,129.5$, 128.8, 117.4, 115.4, 50.9 (-NHCH), 46.5 (benzylic- $\mathrm{CH}_{2}-$ ), 23.6 $\left(-\mathrm{CH}_{3}\right)$; HRMS (ESI) $(\mathrm{M}+\mathrm{H})^{+}$calcd for $\mathrm{C}_{10} \mathrm{H}_{16} \mathrm{~N}_{2}$ : 165.1391, found 165.1358 .

2-((butylamino)methyl)aniline (2f). The compound was obtained as a green solid, yield: $86 \%$; ${ }^{1} \mathrm{H}$ NMR (400 MHz, DMSO$\left.\mathrm{d}_{6}\right) \delta: 7.21(\mathrm{dd}, J=7.32,1.46 \mathrm{~Hz}, 1 \mathrm{H}), 7.07(\mathrm{dt}, J=8.05,1.46 \mathrm{~Hz}$, $1 \mathrm{H}), 6.70(\mathrm{dd}, J=8.05,1.46 \mathrm{~Hz}, 1 \mathrm{H}), 6.57(\mathrm{t}, J=7.32,1.46 \mathrm{~Hz}$, $1 \mathrm{H}), 3.97\left(\mathrm{~s}, 2 \mathrm{H}\right.$, benzylic- $\left.\mathrm{CH}_{2}-\right), 2.87(\mathrm{t}, 2 \mathrm{H}, J=8.05 \mathrm{~Hz}$, $-\mathrm{CH}_{2} \mathrm{CH}_{2} \mathrm{CH}_{2}$ ), 1.62 (q, $2 \mathrm{H}, J=8.05 \mathrm{~Hz},-\mathrm{CH}_{2} \mathrm{CH}_{2} \mathrm{CH}_{2}$ ), 1.30 (sext, $2 \mathrm{H}, J=7.32 \mathrm{~Hz}-\mathrm{CH}_{2} \mathrm{CH}_{2} \mathrm{CH}_{2} \mathrm{CH}_{3}$ ), 0.87 (t, $3 \mathrm{H}, J=7.69 \mathrm{~Hz}$, $\left.-\mathrm{CH}_{3}\right) ;{ }^{13} \mathrm{C}$ NMR (100 MHz, DMSO-d 6 ) $\delta: 147.6,132.1,129.8$, 116.2, 115.9, 115.2, 46.2 (benzylic- $\mathrm{CH}_{2}-$ ), $38.4\left(-\mathrm{NCH}_{2}\right), 27.3$ $\left(-\mathrm{CH}_{2} \mathrm{CH}_{2} \mathrm{CH}_{2}\right), 19.4\left(-\mathrm{CH}_{2} \mathrm{CH}_{3}\right), 13.5\left(-\mathrm{CH}_{3}\right)$; HRMS (ESI) (M + $H)^{+}$calcd for $\mathrm{C}_{11} \mathrm{H}_{18} \mathrm{~N}_{2}$ : 179.1548 , found 179.1537 . 
2-((tert-butylamino)methyl)aniline (2g). The compound was obtained as a dark green solid, yield: $87 \% ;{ }^{1} \mathrm{H}$ NMR $(400 \mathrm{MHz}$, DMSO-d $\left._{6}\right) \delta: 7.00(\mathrm{~d}, J=7.33 \mathrm{~Hz}, 1 \mathrm{H}), 6.93(\mathrm{t}, J=7.79 \mathrm{~Hz}, 1 \mathrm{H})$, $6.60(\mathrm{~d}, J=7.79 \mathrm{~Hz}, 1 \mathrm{H}), 6.48(\mathrm{t}, J=7.33 \mathrm{~Hz}, 1 \mathrm{H}), 3.62(\mathrm{~s}, 2 \mathrm{H}$, benzylic- $\left.\mathrm{CH}_{2}-\right), 1.14\left(\mathrm{~s}, 9 \mathrm{H}, 3 \times \mathrm{CH}_{3}\right) ;{ }^{13} \mathrm{C} \mathrm{NMR}(100 \mathrm{MHz}$, DMSO-d $\left._{6}\right) \delta$ : 147.5, 130.0, 127.5, 116.6, 115.3, 114.3, 51.5 (-NHC), 42.5 (benzylic- $\left.\mathrm{CH}_{2}-\right)$, $27.6\left(-\mathrm{CH}_{3}\right)$; HRMS (ESI) $(\mathrm{M}+\mathrm{H})^{+}$ calcd for $\mathrm{C}_{11} \mathrm{H}_{18} \mathrm{~N}_{2}$ : 179.1548, found 179.1540.

2-((cyclopropylamino)methyl)aniline (2h). The compound was obtained as a light green solid, yield: $87 \% ;{ }^{1} \mathrm{H}$ NMR $(400$ MHz, DMSO-d $\left.{ }_{6}\right) \delta: 6.97(\mathrm{~d}, J=7.32 \mathrm{~Hz}, 1 \mathrm{H}), 6.92(\mathrm{t}, J=7.93$, $7.32 \mathrm{~Hz}, 1 \mathrm{H}), 6.58(\mathrm{~d}, J=7.32 \mathrm{~Hz}, 1 \mathrm{H}), 6.47(\mathrm{t}, J=7.32 \mathrm{~Hz}, 1 \mathrm{H})$, 3.63 (s, 2H, benzylic- $\mathrm{CH}_{2}-$ ), 2.06-2.03 (m, 1H,-NHCH), 0.36$0.31\left(\mathrm{~m}, 2 \mathrm{H}\right.$, cyclopropyl $\left.\mathrm{CH}_{2}\right), 0.25-0.22(\mathrm{~m}, 2 \mathrm{H}$, cyclopropyl $\left.\mathrm{CH}_{2}\right) ;{ }^{13} \mathrm{C}$ NMR $\left(100 \mathrm{MHz}, \mathrm{DMSO}-\mathrm{d}_{6}\right) \delta: 147.2,129.1,127.4$, 123.6, 115.8, 114.6, 51.2 (benzylic- $\mathrm{CH}_{2}-$ ), $30.2(-\mathrm{NHCH}), 5.8$ (cyclopropyl $\mathrm{CH}_{2}$ ).

2-((cyclohexylamino)methyl)aniline (2i). The compound was obtained as a dark green liquid, yield: $84 \%$; ${ }^{1} \mathrm{H}$ NMR $(400 \mathrm{MHz}$, DMSO-d $\left._{6}\right) \delta: 7.21(\mathrm{dd}, J=7.32,1.46 \mathrm{~Hz}, 1 \mathrm{H}), 7.06(\mathrm{t}, J=8.05 \mathrm{~Hz}$, $1 \mathrm{H}), 6.70$ (d, $J=8.05 \mathrm{~Hz}, 1 \mathrm{H}), 6.57$ (t, $J=7.32 \mathrm{~Hz}, 1 \mathrm{H}), 3.96(\mathrm{~s}$, $2 \mathrm{H}$, benzylic- $\left.\mathrm{CH}_{2}-\right)$, 2.99-2.94 (m, $\left.1 \mathrm{H},-\mathrm{NCH}\right), 2.13-2.10(\mathrm{~d}, 2 \mathrm{H})$, 1.77-1.74 (d, 2H), 1.60-1.57 (d, 1H), 1.42-1.33 (m, 2H), 1.25$1.06(\mathrm{~m}, 3 \mathrm{H}) ;{ }^{13} \mathrm{C}$ NMR (100 MHz, DMSO-d $\left.{ }_{6}\right) \delta: 147.4,131.8$, 130.6, 117.6, 116.8, 57.3 (-NHCH), 31.2, 25.2, 24.6.

2-((benzylamino)methyl)aniline (2j). The compound was obtained as a light green solid, yield: $82 \% ;{ }^{1} \mathrm{H}$ NMR $(400 \mathrm{MHz}$, DMSO-d $\left.{ }_{6}\right) \delta: 7.59(\mathrm{~d}, J=7.32 \mathrm{~Hz}, 1 \mathrm{H}), 7.50(\mathrm{~d}, J=7.32 \mathrm{~Hz}, 1 \mathrm{H})$, $7.41-7.34(\mathrm{~m}, 3 \mathrm{H}), 7.22(\mathrm{~d}, J=7.32 \mathrm{~Hz}, 1 \mathrm{H}), 7.06(\mathrm{t}, J=7.32 \mathrm{~Hz}$, $1 \mathrm{H}), 6.72(\mathrm{~d}, J=7.32 \mathrm{~Hz}, 1 \mathrm{H}), 6.68$ (d, $J=7.32 \mathrm{~Hz}, 1 \mathrm{H}) 4.14(\mathrm{~s}$, $2 \mathrm{H}$, benzylic- $\left.\mathrm{CH}_{2 \mathrm{a}}-\right), 4.00\left(\mathrm{~s}, 2 \mathrm{H}\right.$, benzylic- $\left.\mathrm{CH}_{2 \mathrm{~b}}{ }^{-}\right) ;{ }^{13} \mathrm{C}$ NMR $\left(100 \mathrm{MHz} \mathrm{CDCl}_{3}\right) \delta: 146.9,140.2,129.8,128.5,127.5,127.3$, 127.1, 124.2, 118.5, 116.3, 56.8 (benzylic- $\mathrm{CH}_{2 \mathrm{a}}{ }^{-}$), 48.9 (benzylic$\mathrm{CH}_{2 \mathrm{~b}^{-}}$); HRMS (ESI) $(\mathrm{M}+\mathrm{H})^{+}$calcd for $\mathrm{C}_{14} \mathrm{H}_{16} \mathrm{~N}_{2}$ : 213.1391, found 213.1387 .

General procedure for the synthesis 3,4-dihydro-1 $\mathrm{H}$ quinazolin-2-ylidenes (4a-p)

In an oven-dried two-neck RBF, $\alpha$-oxoketene dithioacetal 3a-c (1 equiv.), 1.2 equiv. of 2 -substituted aniline $2 \mathbf{a}-\mathbf{j}, 3.0$ equiv. of $\mathrm{K}_{2} \mathrm{CO}_{3}$ was added in $10 \mathrm{~mL}$ of PEG-400. Reaction mass was heated at $110{ }^{\circ} \mathrm{C}$ for $6-10 \mathrm{~h}$. Progress of reaction was monitored by TLC. The reaction mixture was then cooled and solvent was evaporated under vacuum. Crude was directly purified by column chromatography using silica gel (100: 200 mesh) in 5$10 \%$ ethyl acetate/hexane as eluent to afford the corresponding product. Since PEG is immiscible with diethyl ether, it was washed (two times) with this solvent to remove any unwanted organic impurities and was used successfully in consecutive runs. Although a weight loss of approximately 5\% of PEG was observed from cycle to cycle due to mechanical loss.

\section{Analytical data of 3,4-dihydro-1H-quinazolin-2-ylidenes}

(E)-2-(3,4-dihydroquinazolin-2(1H)-ylidene)-1-(4-fluorophenyl) ethanone (4a). The compound was obtained as light yellow solid, yield: $80 \%$; mp 104-106 ${ }^{\circ} \mathrm{C}$; IR $\left(\nu_{\max } \mathrm{cm}^{-1}\right)\left(\mathrm{CHCl}_{3}\right): 2923$,
2358, 1589; ${ }^{1} \mathrm{H}$ NMR (400 MHz, $\mathrm{CDCl}_{3}$ ) $\delta: 13.23$ (bs, $1 \mathrm{H}, \mathrm{NH}$ ), 7.90 (dd, $J=9.0,3.3 \mathrm{~Hz}, 2 \mathrm{H}), 7.34-7.27$ (m, 1H), 7.14-7.06 (m, $4 \mathrm{H}), 7.00(\mathrm{~d}, J=7.8 \mathrm{~Hz}, 1 \mathrm{H}), 5.68$ (s, 1H, vinylic $\mathrm{CH}), 5.19(\mathrm{~s}, 2 \mathrm{H}$, $\left.-\mathrm{NHCH}_{2}\right) ;{ }^{13} \mathrm{C} \mathrm{NMR}\left(100 \mathrm{MHz}, \mathrm{CDCl}_{3}\right) \delta: 187.2(\mathrm{C}=\mathrm{O}), 165.8$, $164.2,135.7,132.8,129.5,129.0,124.5,123.8,119.3,115.3$, 115.1, 79.1 (vinylic $\mathrm{CH}), 45.7\left(-\mathrm{NHCH}_{2}\right)$; HRMS (ESI) $(\mathrm{M}+\mathrm{H})^{+}$ calcd for $\mathrm{C}_{16} \mathrm{H}_{13} \mathrm{FN}_{2} \mathrm{O}$ : 269.1090, found 269.1085.

(E)-1-(4-fluorophenyl)-2-(3-methyl-3,4-dihydroquinazolin-2(1H)ylidene)ethanone (4b). The compound was obtained as a yellow solid, yield: $90 \%$; mp $138-140{ }^{\circ} \mathrm{C}$; IR $\left(\nu_{\max } \mathrm{cm}^{-1}\right)\left(\mathrm{CHCl}_{3}\right): 2923$, 1594; ${ }^{1} \mathrm{H}$ NMR (400 MHz, DMSO-d 6 ) $\delta: 13.66$ (s, 1H, NH), 7.92 (dd, $J=9.16,3.05 \mathrm{~Hz}, 2 \mathrm{H}), 7.18(\mathrm{t}, J=9.16 \mathrm{~Hz}, 3 \mathrm{H}), 7.07$ (d, $J=$ $6.71 \mathrm{~Hz}, 1 \mathrm{H}), 6.96(\mathrm{t}, J=7.32 \mathrm{~Hz}, 1 \mathrm{H}), 6.91(\mathrm{~d}, J=7.93 \mathrm{~Hz}, 1 \mathrm{H})$, $5.43(\mathrm{~s}, 1 \mathrm{H}$, vinylic $\mathrm{CH}), 4.45\left(\mathrm{~s}, 2 \mathrm{H},-\mathrm{NCH}_{2}\right), 3.03\left(\mathrm{~s}, 3 \mathrm{H},-\mathrm{CH}_{3}\right)$; ${ }^{13} \mathrm{C}$ NMR $\left(100 \mathrm{MHz}, \mathrm{CDCl}_{3}\right) \delta: 184.1(\mathrm{C}=\mathrm{O}), 165.2,162.7,157.4$, 137.5, 134.2, 128.6, 125.4, 122.8, 117.9, 115.3, 115.0, 114.8, 75.9 (vinylic $\mathrm{CH}), 50.7\left(-\mathrm{NCH}_{2}\right), 37.5\left(-\mathrm{CH}_{3}\right)$; HRMS (ESI) $(\mathrm{M}+\mathrm{H})^{+}$ calcd for $\mathrm{C}_{17} \mathrm{H}_{15} \mathrm{FN}_{2} \mathrm{O}$ : 283.1246, found 283.1245.

(E)-2-(3-ethyl-3,4-dihydroquinazolin-2(1H)-ylidene)-1-(4fluorophenyl)ethanone (4c). The compound was obtained as yellow solid, yield: $88 \%$; mp 84-86 ${ }^{\circ} \mathrm{C}$; IR $\left(\nu_{\max } \mathrm{cm}^{-1}\right)\left(\mathrm{CHCl}_{3}\right)$ : 2925, 1593; ${ }^{1} \mathrm{H}$ NMR (400 MHz, DMSO-d 6 ) $\delta: 13.86(\mathrm{~s}, 1 \mathrm{H}, \mathrm{NH}$ ), $7.93(\mathrm{dd}, J=9.16,3.05 \mathrm{~Hz}, 2 \mathrm{H}), 7.21(\mathrm{t}, J=8.85 \mathrm{~Hz}, 3 \mathrm{H}), 7.11(\mathrm{~d}, J$ $=7.32 \mathrm{~Hz}, 1 \mathrm{H}), 6.99(\mathrm{t}, J=7.32 \mathrm{~Hz}, 1 \mathrm{H}), 6.93(\mathrm{~d}, J=7.93 \mathrm{~Hz}, 1 \mathrm{H})$, $5.50(\mathrm{~s}, 1 \mathrm{H}$, vinylic $\mathrm{CH}), 4.49\left(\mathrm{~s}, 2 \mathrm{H},-\mathrm{NCH}_{2}\right), 3.51(\mathrm{q}, 2 \mathrm{H}$, $\left.-\mathrm{CH}_{2} \mathrm{CH}_{3}\right), 1.21\left(\mathrm{t}, 3 \mathrm{H},-\mathrm{CH}_{3}\right) ;{ }^{13} \mathrm{C} \mathrm{NMR}\left(100 \mathrm{MHz}, \mathrm{CDCl}_{3}\right) \delta$ : $183.9(\mathrm{C}=\mathrm{O}), 165.1,162.6,156.7,137.7,134.3,128.6,128.5$, 125.4, 122.8, 117.9, 115.2, 114.9, 114.7, 75.7 (vinylic $\mathrm{CH}$ ), 48.1 $\left(-\mathrm{NCH}_{2}\right), 45.0\left(-\mathrm{CH}_{2} \mathrm{CH}_{3}\right), 11.2\left(-\mathrm{CH}_{3}\right)$; HRMS (ESI) $(\mathrm{M}+\mathrm{H})^{+}$ calcd for $\mathrm{C}_{18} \mathrm{H}_{17} \mathrm{FN}_{2} \mathrm{O}: 297.1403$, found 297.1396.

(E)-1-(4-fluorophenyl)-2-(3-propyl-3,4-dihydroquinazolin-2(1H)ylidene)ethanone (4d). The compound was obtained as light yellow solid, yield: $85 \%$; mp $130-132{ }^{\circ} \mathrm{C}$; IR $\left(\nu_{\max } \mathrm{cm}^{-1}\right)\left(\mathrm{CHCl}_{3}\right)$ : 2924, 1590; ${ }^{1} \mathrm{H}$ NMR (300 MHz, DMSO-d 6 ) $\delta: 13.91$ (s, 1H, NH), $7.93(\mathrm{dd}, J=8.70,3.6 \mathrm{~Hz}, 2 \mathrm{H}), 7.23(\mathrm{t}, J=8.70 \mathrm{~Hz}, 3 \mathrm{H}), 7.12(\mathrm{~d}, J$ $=7.2 \mathrm{~Hz}, 1 \mathrm{H}), 7.01(\mathrm{~d}, J=7.2 \mathrm{~Hz}, 1 \mathrm{H}), 6.96(\mathrm{t}, J=8.1 \mathrm{~Hz}, 1 \mathrm{H})$, $5.50(\mathrm{~s}, 1 \mathrm{H}$, vinylic $\mathrm{CH}), 4.51\left(\mathrm{~s}, 2 \mathrm{H},-\mathrm{NCH}_{2}\right), 3.44(\mathrm{t}, 2 \mathrm{H}$, $\left.-\mathrm{CH}_{2} \mathrm{CH}_{2} \mathrm{CH}_{3}\right), 1.75-1.62\left(\mathrm{~m}, 2 \mathrm{H},-\mathrm{CH}_{2} \mathrm{CH}_{2} \mathrm{CH}_{3}\right), 0.94(\mathrm{t}, 3 \mathrm{H}$, $\left.-\mathrm{CH}_{3}\right) ;{ }^{13} \mathrm{C} \mathrm{NMR}\left(100 \mathrm{MHz}, \mathrm{CDCl}_{3}\right) \delta: 183.8(\mathrm{C}=\mathrm{O}), 165.1,162.6$, 157.1 , 137.7, 134.4, 128.6, 125.3, 122.8, 117.9, 115.2, 115.0, 114.7, 75.9 (vinylic $\mathrm{CH}), 52.1\left(-\mathrm{CH}_{2} \mathrm{CH}_{2} \mathrm{CH}_{3}\right), 49.0\left(-\mathrm{NCH}_{2}\right), 19.8$ $\left(-\mathrm{CH}_{2} \mathrm{CH}_{3}\right), 11.3\left(-\mathrm{CH}_{3}\right)$; HRMS (ESI) $(\mathrm{M}+\mathrm{H})^{+}$calcd for $\mathrm{C}_{19} \mathrm{H}_{19} \mathrm{FN}_{2} \mathrm{O}$ : 311.1559, found 311.1553.

(E)-1-(4-fluorophenyl)-2-(3-isopropyl-3,4-dihydroquinazolin2(1H)-ylidene)ethanone (4e). The compound was obtained as a light yellow viscous material; yield: $82 \%$; IR $\left(\nu_{\max } \mathrm{cm}^{-1}\right)$ $\left(\mathrm{CHCl}_{3}\right): 2925,1584 ;{ }^{1} \mathrm{H}$ NMR (400 MHz, DMSO-d $\left.{ }_{6}\right) \delta: 13.75$ (s, $1 \mathrm{H},-N \mathrm{H}), 8.12(\mathrm{dd}, J=8.54,3.0 \mathrm{~Hz}, 2 \mathrm{H}), 7.44(\mathrm{t}, J=8.54 \mathrm{~Hz}$, $2 \mathrm{H}), 7.34-7.23(\mathrm{~m}, 3 \mathrm{H}), 7.18(\mathrm{~d}, J=7.32 \mathrm{~Hz}, 1 \mathrm{H}), 5.10(\mathrm{~s}, 1 \mathrm{H}$, vinylic $\mathrm{CH}), 4.84\left(\mathrm{~s}, 2 \mathrm{H},-\mathrm{NCH}_{2}\right), 4.24(\mathrm{q}, J=6.41 \mathrm{~Hz}, 1 \mathrm{H},-\mathrm{NCH})$, $1.25\left(\mathrm{~d}, J=6.41 \mathrm{~Hz}, 6 \mathrm{H}, 2 \times \mathrm{CH}_{3}\right) ;{ }^{13} \mathrm{C}$ NMR (100 MHz, DMSO$\left.\mathrm{d}_{6}\right) \delta: 191.1(\mathrm{C}=\mathrm{O}), 166.9,164.4,156.5,135.3,133.1,131.7$, 131.4, 128.9, 126.8, 126.5, 117.6, 116.1, 115.9, 115.7, 79.0 (vinylic $\left.\mathrm{CH}), 52.3(-\mathrm{NCH}), 41.5\left(-\mathrm{NCH}_{2}\right), 18.6-\mathrm{CH}_{3}\right)$; HRMS (ESI) (M + $\mathrm{H})^{+}$calcd for $\mathrm{C}_{19} \mathrm{H}_{19} \mathrm{~F}_{2} \mathrm{O}$ : 311.1559, found 311.1553; ESI-MS $(\mathrm{m} / \mathrm{z})$. 
(E)-2-(3-butyl-3,4-dihydroquinazolin-2(1H)-ylidene)-1-(4fluorophenyl)ethanone (4f). The compound was obtained as a light yellow solid, yield: $92 \%$; mp $108-110{ }^{\circ} \mathrm{C}$; IR $\left(\nu_{\max } \mathrm{cm}^{-1}\right)$ $\left(\mathrm{CHCl}_{3}\right): 2924,1590 ;{ }^{1} \mathrm{H}$ NMR (400 MHz, DMSO-d $\left.{ }_{6}\right) \delta: 13.89(\mathrm{~s}$, $1 \mathrm{H}, N \mathrm{H}), 7.93(\mathrm{dd}, J=9.0,3.3 \mathrm{~Hz}, 2 \mathrm{H}), 7.23(\mathrm{t}, J=9.0 \mathrm{~Hz}, 3 \mathrm{H})$, $7.13(\mathrm{t}, J=7.5 \mathrm{~Hz}, 1 \mathrm{H}), 7.01(\mathrm{~d}, J=7.5 \mathrm{~Hz}, 1 \mathrm{H}), 6.96(\mathrm{t}, J=7.8 \mathrm{~Hz}$, $1 \mathrm{H}), 5.49(\mathrm{~s}, 1 \mathrm{H}$, vinylic $\mathrm{CH}), 4.51\left(\mathrm{~s}, 2 \mathrm{H},-\mathrm{NCH}_{2}\right), 3.47(\mathrm{t}, 2 \mathrm{H}$, $\mathrm{CH}_{2} \mathrm{CH}_{2} \mathrm{CH}_{2} \mathrm{CH}_{3}$ ), 1.65 (q, 2H, $\left.\mathrm{CH}_{2} \mathrm{CH}_{3}\right), 1.44-1.32(\mathrm{~m}, 2 \mathrm{H}$, $\left.-\mathrm{CH}_{2} \mathrm{CH}_{2} \mathrm{CH}_{3}\right), 0.94\left(\mathrm{t}, 3 \mathrm{H},-\mathrm{CH}_{3}\right) ;{ }^{13} \mathrm{C} \mathrm{NMR}\left(100 \mathrm{MHz}, \mathrm{CDCl}_{3}\right) \delta$ : $183.7(\mathrm{C}=\mathrm{O}), 165.1,162.6,157.0,137.7,134.5,128.6,125.3$, 122.8, 117.9, 115.2, 115.0, 114.7, 75.8 (vinylic $\mathrm{CH}), 50.3\left(-\mathrm{CH}_{2}-\right.$ $\left.\mathrm{CH}_{2} \mathrm{CH}_{2} \mathrm{CH}_{3}\right), \quad 48.9 \quad\left(-\mathrm{NCH}_{2}\right), \quad 28.4 \quad\left(-\mathrm{CH}_{2} \mathrm{CH}_{2} \mathrm{CH}_{3}\right), \quad 20.1$ $\left(-\mathrm{CH}_{2} \mathrm{CH}_{3}\right), 13.8\left(-\mathrm{CH}_{3}\right)$; HRMS (ESI) $(\mathrm{M}+\mathrm{H})^{+}$calcd for $\mathrm{C}_{20} \mathrm{H}_{21} \mathrm{FN}_{2} \mathrm{O}$ : 325.1716, found 325.1724.

(E)-2-(3-(tert-butyl)-3,4-dihydroquinazolin-2(1H)-ylidene)-1-(4fluorophenyl)ethanone $\mathbf{( 4 g )}$. The compound was obtained as a light yellow solid, yield: $86 \%$; mp $106-108{ }^{\circ} \mathrm{C}$; IR $\left(\nu_{\max } \mathrm{cm}^{-1}\right)$ $\left(\mathrm{CHCl}_{3}\right): 2925,1581 ;{ }^{1} \mathrm{H}$ NMR (400 MHz, DMSO-d $\left.{ }_{6}\right) \delta: 14.3$ (s, $1 \mathrm{H}, N \mathrm{H}), 7.83(\mathrm{dd}, J=9.16,3.66 \mathrm{~Hz}, 2 \mathrm{H}), 7.23-7.17(\mathrm{~m}, 4 \mathrm{H}), 6.97$ $(\mathrm{t}, J=7.32 \mathrm{~Hz}, 1 \mathrm{H}), 6.93(\mathrm{~d}, J=7.93 \mathrm{~Hz}, 1 \mathrm{H}), 5.60(\mathrm{~s}, 1 \mathrm{H}$, vinylic $\mathrm{CH}), 4.34\left(\mathrm{~s}, 2 \mathrm{H},-\mathrm{NCH}_{2}\right), 1.51\left(\mathrm{~s}, 9 \mathrm{H}, 3 \times \mathrm{CH}_{3}\right) ;{ }^{13} \mathrm{C} \mathrm{NMR}(100$ MHz, DMSO-d 6 ) $\delta: 182.9(\mathrm{C}=\mathrm{O}), 159.0,137.8,135.4,128.6$, 124.6, 122.9, 121.6, 115.1, 114.8, 82.4 (vinylic $\mathrm{CH}), 57.1\left(-\mathrm{NCH}_{2}\right)$, $43.9\left(-\mathrm{C}\left(\mathrm{CH}_{3}\right)_{3}\right), 29.7\left(-\mathrm{CH}_{3}\right)$; HRMS (ESI) $(\mathrm{M}+\mathrm{H})^{+}$calcd for $\mathrm{C}_{20} \mathrm{H}_{21} \mathrm{FN}_{2} \mathrm{O}: 325.1716$, found 325.1716.

(E)-2-(3-cyclopropyl-3,4-dihydroquinazolin-2(1H)-ylidene)-1(4-fluorophenyl) ethanone (4h). The compound was obtained as a yellow viscous material; yield: $70 \%$; IR $\left(\nu_{\max } \mathrm{cm}^{-1}\right)\left(\mathrm{CHCl}_{3}\right)$ : 2924, 1595; ${ }^{1} \mathrm{H}$ NMR (400 MHz, DMSO-d 6 ) $\delta: 13.5(\mathrm{~s}, 1 \mathrm{H}, \mathrm{NH})$, $7.87(\mathrm{dd}, J=8.79,2.93 \mathrm{~Hz}, 2 \mathrm{H}), 7.18(\mathrm{t}, J=7.32 \mathrm{~Hz}, 1 \mathrm{H}), 7.06(\mathrm{t}, J$ $=8.79 \mathrm{~Hz}, 2 \mathrm{H}), 7.00-6.95(\mathrm{~m}, 2 \mathrm{H}), 6.91(\mathrm{~d}, J=8.05 \mathrm{~Hz}, 1 \mathrm{H}), 5.82$ $(\mathrm{s}, 1 \mathrm{H}$, vinylic $\mathrm{CH}), 4.37\left(\mathrm{~s}, 2 \mathrm{H},-\mathrm{NCH}_{2}\right), 2.60-2.55(\mathrm{~m}, 1 \mathrm{H}$, -NCH), 1.00-0.96 (q, 2H, cyclopropyl $\left.\mathrm{CH}_{2}\right), 0.81-0.79(\mathrm{~m}, 2 \mathrm{H}$, cyclopropyl $\left.\mathrm{CH}_{2}\right) ;{ }^{13} \mathrm{C} \mathrm{NMR}\left(100 \mathrm{MHz}, \mathrm{CDCl}_{3}\right) \delta: 184.2(\mathrm{C}=\mathrm{O})$, 165.2, 162.7, 159.2, 137.4, 134.1, 128.7, 125.3, 122.8, 119.2, 115.1, 115.0, 114.8, 77.9 (vinylic $\mathrm{CH}), 48.7\left(-\mathrm{NCH}_{2}\right), 30.5(-\mathrm{NCH})$, 9.0 (cyclopropyl $\left.\mathrm{CH}_{2}\right)$; HRMS (ESI) $(\mathrm{M}+\mathrm{H})^{+}$calcd for $\mathrm{C}_{19} \mathrm{H}_{17} \mathrm{FN}_{2} \mathrm{O}: 309.1403$, found 309.1405.

(E)-2-(3-cyclohexyl-3,4-dihydroquinazolin-2(1H)-ylidene)-1(4-fluorophenyl)ethanone (4i). The compound was obtained as a light yellow solid, yield: $71 \%$; mp $116-118{ }^{\circ} \mathrm{C}$; IR $\left(\nu_{\max } \mathrm{cm}^{-1}\right)$ $\left(\mathrm{CHCl}_{3}\right): 2927,2360,1584 ;{ }^{1} \mathrm{H} \mathrm{NMR}\left(400 \mathrm{MHz}, \mathrm{CDCl}_{3}\right) \delta: 13.97$ (s, $1 \mathrm{H}, N \mathrm{H}), 7.82(\mathrm{dd}, J=8.79,2.93 \mathrm{~Hz}, 2 \mathrm{H}), 7.24-7.17(\mathrm{~m}, 3 \mathrm{H}), 7.06$ $(\mathrm{t}, J=8.79 \mathrm{~Hz}, 1 \mathrm{H}), 7.00-6.93(\mathrm{~m}, 2 \mathrm{H}), 5.38(\mathrm{~s}, 1 \mathrm{H}$, vinylic $\mathrm{CH})$, $4.30\left(\mathrm{~s}, 2 \mathrm{H},-\mathrm{NCH}_{2}\right), 3.80-3.74(\mathrm{~m}, 1 \mathrm{H},-\mathrm{NCH}), 1.76-1.60(\mathrm{~m}$, $6 \mathrm{H}), 1.47-1.38(\mathrm{~m}, 2 \mathrm{H}), 1.26-1.15(\mathrm{~m}, 2 \mathrm{H}) ;{ }^{13} \mathrm{C}$ NMR $(100 \mathrm{MHz}$, $\left.\mathrm{CDCl}_{3}\right) \delta: 183.9(\mathrm{C}=\mathrm{O}), 165.1,162.6,157.8,137.9,134.9,128.6$, 125.2, 122.8, 118.7, 115.0, 114.8, 75.9 (vinylic $\mathrm{CH}), 56.5(-\mathrm{NCH})$, $41.7\left(-\mathrm{NCH}_{2}\right), 29.5\left(-\mathrm{NCHCH}_{2}\right), 25.7\left(-\mathrm{NCHCH}_{2} \mathrm{CH}_{2} \mathrm{CH}_{2}\right), 25.4$ $\left(-\mathrm{NCHCH}_{2} \mathrm{CH}_{2} \mathrm{CH}_{2}\right)$; HRMS (ESI) $(\mathrm{M}+\mathrm{H})^{+}$calcd for $\mathrm{C}_{22} \mathrm{H}_{23} \mathrm{FN}_{2} \mathrm{O}: 351.1872$, found 351.1892.

(E)-2-(1-benzyl-3,4-dihydroquinazolin-2(1H)-ylidene)-1-(4fluorophenyl)ethanone $(\mathbf{4 j})$. The compound was obtained as a light yellow viscous material; yield: 68\%; IR $\left(\nu_{\max } \mathrm{cm}^{-1}\right)$ $\left(\mathrm{CHCl}_{3}\right): 2937,1592 ;{ }^{1} \mathrm{H} \mathrm{NMR}\left(400 \mathrm{MHz}, \mathrm{CDCl}_{3}\right) \delta: 13.8(\mathrm{~s}, 1 \mathrm{H}$, $N \mathrm{H}), 7.76(\mathrm{dd}, J=8.79,2.93 \mathrm{~Hz}, 2 \mathrm{H}), 7.39-7.31(\mathrm{~m}, 6 \mathrm{H}), 7.21(\mathrm{t}, J$
$=7.32 \mathrm{~Hz}, 1 \mathrm{H}), 7.03-6.96(\mathrm{~m}, 3 \mathrm{H}), 6.92(\mathrm{t}, J=7.32 \mathrm{~Hz}, 1 \mathrm{H}), 5.46$ (s, 1H, vinylic $\mathrm{CH}), 4.61\left(\mathrm{~s}, 2 \mathrm{H},-\mathrm{NCH}_{2, \text { ring }}\right), 4.41(\mathrm{~s}, 2 \mathrm{H}$, $\left.-\mathrm{NHCH}_{2}\right) ;{ }^{13} \mathrm{C} \mathrm{NMR}\left(100 \mathrm{MHz}, \mathrm{CDCl}_{3}\right) \delta: 184.3(\mathrm{C}=\mathrm{O}), 165.2$, 162.7 , 157.5, 137.4, 134.9, 134.2, 130.3, 129.0, 128.6, 127.9, $126.8,125.4,123.0,117.9,115.4,115.0,114.7,76.3$ (vinylic $\mathrm{CH}$ ), $48.7\left(-\mathrm{NCH}_{2}\right), 44.5\left(-\mathrm{NCH}_{2, \text { ring }}\right)$; HRMS (ESI) $(\mathrm{M}+\mathrm{H})^{+}$calcd for $\mathrm{C}_{23} \mathrm{H}_{19} \mathrm{FN}_{2} \mathrm{O}: 359.1559$, found 359.1583.

(E)-2-(3-cyclohexyl-3,4-dihydroquinazolin-2(1H)-ylidene)-1(4-fluoro-2-methoxy phenyl)ethanone (4k). The compound was obtained as a light yellow solid, yield: $65 \%$; mp $148-150{ }^{\circ} \mathrm{C}$; IR $\left(\nu_{\max } \mathrm{cm}^{-1}\right)\left(\mathrm{CHCl}_{3}\right): 2937,1580 ;{ }^{1} \mathrm{H} \mathrm{NMR}\left(400 \mathrm{MHz}, \mathrm{CDCl}_{3}\right) \delta$ : $13.86(\mathrm{~s}, 1 \mathrm{H}, N \mathrm{H}), 7.68(\mathrm{t}, J=8.54 \mathrm{~Hz}, 1 \mathrm{H}), 7.18(\mathrm{t}, J=6.71 \mathrm{~Hz}$, $1 \mathrm{H}), 6.98-6.90(\mathrm{~m}, 3 \mathrm{H}), 6.73-6.61(\mathrm{~m}, 2 \mathrm{H}), 5.47$ (s, $1 \mathrm{H}$, vinylic $\mathrm{CH}), 4.28\left(\mathrm{~s}, 2 \mathrm{H},-\mathrm{NCH}_{2}\right), 3.85\left(\mathrm{~s}, 3 \mathrm{H}, \mathrm{Ar}-\mathrm{OCH}_{3}\right), 3.73-3.68(\mathrm{~m}$, $1 \mathrm{H},-\mathrm{NCH}), 1.72-1.54(\mathrm{~m}, 6 \mathrm{H}), 1.40-1.30(\mathrm{~m}, 2 \mathrm{H}), 1.26-1.13(\mathrm{~m}$, $2 \mathrm{H}) ;{ }^{13} \mathrm{C} \mathrm{NMR}\left(100 \mathrm{MHz}, \mathrm{CDCl}_{3}\right) \delta: 183.7(\mathrm{C}=\mathrm{O}), 165.1,162.7$, $158.2,157.5,134.9,131.8,128.5$, 128.1, 125.2, 122.6, 118.6, 115.1, 107.0, 106.7, 99.4, 99.1, 81.0 (vinylic $\mathrm{CH}), 56.4(-\mathrm{NCH})$, $\begin{array}{llllll}55.8 & \left(\mathrm{Ar}-\mathrm{OCH}_{3}\right), & 41.6 & \left(-\mathrm{NCH}_{2}\right), & 29.5 & \left(-\mathrm{NCHCH}_{2}\right), \quad 25.7\end{array}$ $\left(-\mathrm{NCHCH}_{2} \mathrm{CH}_{2} \mathrm{CH}_{2}\right), 25.4\left(-\mathrm{NCHCH}_{2} \mathrm{CH}_{2} \mathrm{CH}_{2}\right) ; \mathrm{HRMS}$ (ESI) (M + $\mathrm{H})^{+}$calcd for $\mathrm{C}_{21} \mathrm{H}_{23} \mathrm{FN}_{2} \mathrm{O}_{2}: 381.1978$, found 381.1975.

(E)-2-(3-butyl-3,4-dihydroquinazolin-2(1H)-ylidene)-1-(4-fluoro-2methoxyphenyl) ethanone (41). The compound was obtained as a light brown solid, yield: $82 \%$; mp $102-104{ }^{\circ} \mathrm{C}$; IR $\left(\nu_{\max } \mathrm{cm}^{-1}\right)$ $\left(\mathrm{CHCl}_{3}\right): 2924,1584 ;{ }^{1} \mathrm{H} \mathrm{NMR}\left(400 \mathrm{MHz}, \mathrm{CDCl}_{3}\right) \delta: 13.66(\mathrm{~s}, 1 \mathrm{H}$, $N \mathrm{H}), 7.65(\mathrm{t}, J=7.34 \mathrm{~Hz}, 1 \mathrm{H}), 7.22-7.14(\mathrm{~m}, 1 \mathrm{H}), 6.94-6.88(\mathrm{~m}$, $3 \mathrm{H})$, 6.65-6.60 (m, 2H), $5.36(\mathrm{~s}, 1 \mathrm{H}$, vinylic $\mathrm{CH}), 4.40(\mathrm{~s}, 2 \mathrm{H}$, $\left.-\mathrm{NCH}_{2}\right), 3.83\left(\mathrm{~s}, 3 \mathrm{H}, \mathrm{Ar}-\mathrm{OCH}_{3}\right), 3.28\left(\mathrm{t}, J=7.34 \mathrm{~Hz}, 2 \mathrm{H},-\mathrm{CH}_{2}-\right.$ $\mathrm{CH}_{2} \mathrm{CH}_{2} \mathrm{CH}_{3}$ ), 1.75-1.67 (m, 2H, $\left.\mathrm{CH}_{2} \mathrm{CH}_{2} \mathrm{CH}_{2} \mathrm{CH}_{3}\right), 1.40-1.34$ $\left(\mathrm{m}, 2 \mathrm{H}, \mathrm{CH}_{2} \mathrm{CH}_{3}\right), 0.98-0.91\left(\mathrm{~m}, 3 \mathrm{H},-\mathrm{CH}_{3}\right) ;{ }^{13} \mathrm{C} \mathrm{NMR}(100 \mathrm{MHz}$, $\left.\mathrm{CDCl}_{3}\right) \delta: 183.7(\mathrm{C}=\mathrm{O}), 158.1,156.6,153.6,134.5,131.0,128.6$, 125.2, 122.6, 115.3, 107.0, 106.7, 99.4, 99.1, 80.9 (vinylic $\mathrm{CH}$ ), $55.8\left(\mathrm{Ar}-\mathrm{OCH}_{3}\right), 50.4\left(-\mathrm{NCH}_{2, \text { ring }}\right), 29.6\left(-\mathrm{CH}_{2} \mathrm{CH}_{2} \mathrm{CH}_{2} \mathrm{CH}_{3}\right), 20.1$ $\left(-\mathrm{CH}_{2} \mathrm{CH}_{3}\right), 13.8\left(-\mathrm{CH}_{3}\right)$; HRMS (ESI) $(\mathrm{M}+\mathrm{H})^{+}$calcd for $\mathrm{C}_{21} \mathrm{H}_{23} \mathrm{FN}_{2} \mathrm{O}_{2}$ : 355.1822, found 355.1820.

(E)-2-(3-cyclopropyl-3,4-dihydroquinazolin-2(1H)-ylidene)-1(4-fluoro-2-methoxy phenyl)ethanone (4m). The compound was obtained as a light brown solid, mp 108-110 ${ }^{\circ} \mathrm{C}$; IR $\left(\nu_{\max } \mathrm{cm}^{-1}\right)$ $\left(\mathrm{CHCl}_{3}\right)$ : 2928, 1588; ${ }^{1} \mathrm{H}$ NMR $\left(400 \mathrm{MHz}\right.$, yield: $\left.82 \% ; \mathrm{CDCl}_{3}\right) \delta$ : $13.53(\mathrm{~s}, 1 \mathrm{H}, N \mathrm{H}), 7.67(\mathrm{t}, J=8.70 \mathrm{~Hz}, 1 \mathrm{H}), 7.61(\mathrm{t}, J=7.79 \mathrm{~Hz}$, $1 \mathrm{H}), 6.96(\mathrm{t}, J=7.33 \mathrm{~Hz}, 1 \mathrm{H}) 6.91(\mathrm{~d}, J=7.79 \mathrm{~Hz}, 1 \mathrm{H}), 6.88(\mathrm{~d}, J=$ $7.79 \mathrm{~Hz}, 1 \mathrm{H}), 6.67(\mathrm{dd}, J=8.70,2.29 \mathrm{~Hz}, 1 \mathrm{H}), 6.65-6.61(\mathrm{~m}, 1 \mathrm{H})$, $5.84(\mathrm{~s}, 1 \mathrm{H}$, vinylic $\mathrm{CH}), 4.34\left(\mathrm{~s}, 2 \mathrm{H},-\mathrm{NCH}_{2}\right), 3.84(\mathrm{~s}, 3 \mathrm{H}, \mathrm{Ar}-$ $\left.\mathrm{OCH}_{3}\right), 2.52-2.45(\mathrm{~m}, 1 \mathrm{H},-\mathrm{NCH}), 0.92-0.87$ (q, 2H, cyclopropyl $\mathrm{CH}_{2}$ ), 0.79-0.75 (m, 2H, cyclopropyl $\left.\mathrm{CH}_{2}\right) ;{ }^{13} \mathrm{C}$ NMR $(100 \mathrm{MHz}$, $\left.\mathrm{CDCl}_{3}\right) \delta: 184.4(\mathrm{C}=\mathrm{O}), 165.2,162.8,158.8,158.3,134.3,131.1$, 128.5, 127.8, 125.3, 122.6, 119.2, 115.1, 106.9, 99.4, 83.0 (vinylic $\mathrm{CH}), 55.7\left(\mathrm{Ar}-\mathrm{OCH}_{3}\right), 48.7\left(-\mathrm{NHCH}_{2}\right), 30.5(-\mathrm{NCH}), 8.8$ (cyclopropyl $\left.\mathrm{CH}_{2}\right)$; HRMS (ESI) $(\mathrm{M}+\mathrm{H})^{+}$calcd for $\mathrm{C}_{20} \mathrm{H}_{19} \mathrm{FN}_{2} \mathrm{O}_{2}$ : 339.1509, found 339.1498.

(E)-2-(3-butyl-3,4-dihydroquinazolin-2(1H)-ylidene)-1-(4(trifluoromethyl)phenyl) ethanone (4n). The compound was obtained as a light yellow solid, yield: $80 \%$; mp $114-116{ }^{\circ} \mathrm{C}$; IR $\left(\nu_{\max } \mathrm{cm}^{-1}\right)\left(\mathrm{CHCl}_{3}\right): 2925,1586 ;{ }^{1} \mathrm{H} \mathrm{NMR}\left(400 \mathrm{MHz}, \mathrm{CDCl}_{3}\right) \delta$ : $13.76(\mathrm{~s}, 1 \mathrm{H}, N \mathrm{H}), 7.90(\mathrm{~d}, J=7.79 \mathrm{~Hz}, 2 \mathrm{H}), 7.63(\mathrm{~d}, J=7.79 \mathrm{~Hz}$, 2H), 7.24-7.18 (m, 1H), 6.98-6.92 (m, 3H), 5.34 (s, 1H, vinylic $\mathrm{CH}), 4.45\left(\mathrm{~s}, 2 \mathrm{H},-\mathrm{NCH}_{2}\right), 3.37(\mathrm{t}, J=7.33 \mathrm{~Hz}, 3 \mathrm{H}$, 
$\left.-\mathrm{CH}_{2} \mathrm{CH}_{2} \mathrm{CH}_{2} \mathrm{CH}_{3}\right), 1.72\left(\mathrm{q}, J=7.33 \mathrm{~Hz}, 2 \mathrm{H}, \mathrm{CH}_{2} \mathrm{CH}_{2} \mathrm{CH}_{3}\right), 1.44-$ 1.39 (q, 2H, $\left.-\mathrm{CH}_{2} \mathrm{CH}_{3}\right), 0.98\left(\mathrm{t}, J=7.33 \mathrm{~Hz}, 3 \mathrm{H}, \mathrm{CH}_{3}\right) ;{ }^{13} \mathrm{C} \mathrm{NMR}$ $\left(100 \mathrm{MHz}, \mathrm{CDCl}_{3}\right) \delta: 183.3(\mathrm{C}=\mathrm{O}), 157.2,145.1,134.2,128.8$, 126.8, 125.4, 125.1, 123.1, 117.8, 115.4 (vinylic $\mathrm{CH}$ ), 50.4 $\left(-\mathrm{NCH}_{2}\right), 49.0\left(-\mathrm{CH}_{2} \mathrm{CH}_{2} \mathrm{CH}_{2} \mathrm{CH}_{3}\right), 28.5\left(\mathrm{CH}_{2} \mathrm{CH}_{2} \mathrm{CH}_{3}\right), 20.1$ $\left(-\mathrm{CH}_{2} \mathrm{CH}_{3}\right), 13.8\left(\mathrm{CH}_{3}\right)$; HRMS (ESI) $(\mathrm{M}+\mathrm{H})^{+}$calcd for $\mathrm{C}_{21} \mathrm{H}_{21} \mathrm{~F}_{3} \mathrm{~N}_{2} \mathrm{O}$ : 375.1684, found 375.1680.

(E)-2-(3-cyclopropyl-3,4-dihydroquinazolin-2(1H)-ylidene)1-(4-(trifluoromethyl) phenyl)ethanone (4o). The compound was obtained as a fluorescent yellow solid; yield: $65 \%$; mp 123$125{ }^{\circ} \mathrm{C}$; IR $\left(\nu_{\max } \mathrm{cm}^{-1}\right)\left(\mathrm{CHCl}_{3}\right): 2923,1590 ;{ }^{1} \mathrm{H}$ NMR $(400 \mathrm{MHz}$, $\left.\mathrm{CDCl}_{3}\right) \delta: 13.6(\mathrm{bs}, 1 \mathrm{H}, N \mathrm{H}), 7.95(\mathrm{~d}, J=8.24 \mathrm{~Hz}, 2 \mathrm{H}), 7.64(\mathrm{~d}, J=$ $8.24 \mathrm{~Hz}, 2 \mathrm{H}), 7.20(\mathrm{t}, J=7.79 \mathrm{~Hz}, 1 \mathrm{H}), 7.01-6.92(\mathrm{~m}, 3 \mathrm{H}), 5.86(\mathrm{~s}$, $1 \mathrm{H}$, vinylic $\mathrm{CH}), 4.41\left(\mathrm{~s}, 2 \mathrm{H},-\mathrm{NCH}_{2}\right), 2.60(\mathrm{~m}, 1 \mathrm{H},-\mathrm{NCH}), 1.02-$ $0.97\left(\mathrm{q}, J=5.50 \mathrm{~Hz}, 2 \mathrm{H}\right.$, cyclopropyl $\left.\mathrm{CH}_{2}\right), 0.83-0.79(\mathrm{~m}, 2 \mathrm{H}$, cyclopropyl $\left.\mathrm{CH}_{2}\right) ;{ }^{13} \mathrm{C}$ NMR $\left(100 \mathrm{MHz}, \mathrm{CDCl}_{3}\right) \delta: 183.7(\mathrm{C}=\mathrm{O})$, 159.4, 144.6, 133.9, 128.7, 126.9, 125.4, 125.1, 123.1, 119.1, 115.3, 78.7 (vinylic $\mathrm{CH}), 48.7\left(-\mathrm{NCH}_{2}\right), 30.6(-\mathrm{NCH}), 9.0$ (cyclopropyl $\left.\mathrm{CH}_{2}\right)$; HRMS (ESI) $(\mathrm{M}+\mathrm{H})^{+}$calcd for $\mathrm{C}_{20} \mathrm{H}_{17} \mathrm{~F}_{3} \mathrm{~N}_{2} \mathrm{O}$ : 359.1371 , found 359.1394 .

(E)-2-(3-cyclohexyl-3,4-dihydroquinazolin-2(1H)-ylidene)-1(4-(trifluoromethyl) phenyl)ethanone (4p). The compound was obtained as a light yellow viscous material; yield: 66\%; IR $\left(\nu_{\max } \mathrm{cm}^{-1}\right)\left(\mathrm{CHCl}_{3}\right): 2928,1584 ;{ }^{1} \mathrm{H} \mathrm{NMR}\left(400 \mathrm{MHz}, \mathrm{CDCl}_{3}\right) \delta$ : $13.96(\mathrm{~s}, 1 \mathrm{H}, N \mathrm{H}), 7.91(\mathrm{~d}, J=8.39 \mathrm{~Hz}, 2 \mathrm{H}), 7.64(\mathrm{~d}, J=8.39 \mathrm{~Hz}$, 2H), 7.26-7.18 (m, 1H), 7.00-6.94 (m, 3H), 5.41 (s, 1H, vinylic $\mathrm{CH}), 4.32\left(\mathrm{~s}, 2 \mathrm{H},-\mathrm{NCH}_{2}\right), 3.81-3.74(\mathrm{~m}, 1 \mathrm{H},-\mathrm{NCH}), 1.92-1.84$ $(\mathrm{m}, 3 \mathrm{H}), 1.64-1.60(\mathrm{~m}, 1 \mathrm{H}), 1.43-1.39(\mathrm{~m}, 2 \mathrm{H}), 1.26-1.21(\mathrm{~m}$, $4 \mathrm{H}) ;{ }^{13} \mathrm{C} \mathrm{NMR}\left(100 \mathrm{MHz}, \mathrm{CDCl}_{3}\right) \delta: 183.4(\mathrm{C}=\mathrm{O}), 157.9,145.1$, $134.6,131.5,131.1,128.7,126.8,125.3$, 125.1, 123.1, 118.5, 115.1, 76.9 (vinylic $\mathrm{CH}), 56.6(-\mathrm{NCH}), 41.8\left(-\mathrm{NCH}_{2}\right), 29.5$ $\left(-\mathrm{NCHCH}_{2}\right), 25.7\left(-\mathrm{NCHCH}_{2} \mathrm{CH}_{2} \mathrm{CH}_{2}\right), 25.3\left(-\mathrm{NCHCH}_{2} \mathrm{CH}_{2}-\right.$ $\left.\mathrm{CH}_{2}\right)$; HRMS (ESI) $(\mathrm{M}+\mathrm{H})^{+}$calcd for $\mathrm{C}_{23} \mathrm{H}_{23} \mathrm{~F}_{3} \mathrm{~N}_{2} \mathrm{O}: 401.1840$, found 401.1832 .

\section{Conflicts of interest}

There are no conflicts to declare.

\section{Acknowledgements}

The authors are thankful to SERB, Department of Science \& Technology, INDIA for providing financial support and USIC, University of Delhi for providing instrumentation facilities.

\section{Notes and references}

1 (a) H. A. Burris III, Oncologist, 2004, 9, 10; (b) T. P. Selvam and P. V. Kumar, Res. Pharm., 2011, 1, 1; (c) S. L. Schreiber, Nat. Chem. Biol., 2005, 1, 64; (d) I. Collins, P. Workman, Nat. Chem. Biol., 2006, 2, 689; (e) A. Foster, H. A. Coffrey, M. J. Morin and F. Rastinejad, Science, 1999, 286, 2507.

2 (a) P. A. Ple, T. P. Green, L. F. Hennequin, J. Curwen, M. Fennell, J. Allen, C. Lambertvan der Brempt and G. Costello, J. Med. Chem., 2004, 47, 871; (b) A. M. Alafeefy, A. A. Kadi, O. A. Al-Deeb, K. E. H. El-Tahir and N. A. AlJaber, Eur. J. Med. Chem., 2010, 45, 4947; (c) V. Alagarsamy,
V. Muthukumar, N. Pavalarani, P. Vasanthanathan and R. Revathi, Biol. Pharm. Bull., 2003, 26, 557; (d) K. M. Amin, M. M. Kamel, M. M. Anwar, M. Khedr and Y. M. Syam, Eur. J. Med. Chem., 2010, 45, 2117.

3 (a) A. J. Barker, E.P. Patent, 0635498, 2001; (b) K. Scherlach, H. W. Nuetzmann, V. Schroeckh, H. M. Dahse, A. A. Brakhage and C. Hertweck, Angew. Chem., Int. Ed., 2011, 50, 9843; (c) P. Thanigaimalai, V. K. Sharma, K. C. Lee, C. Y. Yun, Y. Kim and S. H. Jung, Bioorg. Med. Chem. Lett., 2010, 20, 4771; (d) L. A. Doyle and D. D. Ross, Oncogene, 2003, 22, 7340.

4 (a) P. M. Chandrika, T. Yakaiah, A. R. Ram Rao, B. Narsaiah, N. C. Reddy, V. Sridhar and J. V. Rao, Eur. J. Med. Chem., 2008, 43, 846; (b) A. Baba, N. Kawamura, H. Makino, Y. Ohta, S. Taketomi and S. Sohda, J. Med. Chem., 1996, 39, 5176.

5 J. Rosenberg, F. Gustafsson, S. Galatius and P. R. Hilderbrandt, Cardiovasc. Drugs Ther., 2005, 19, 301.

6 V. G. Ugale and S. B. Bari, Eur. J. Med. Chem., 2014, 80, 447. 7 (a) T. Mohamed, M. K. Mann and P. P. N. Rao, RSC Adv., 2017, 7, 22360; (b) R. Subramaniam, G. Rao and S. P. P. Nagesh, Pharm. Sin., 2011, 2, 146.

8 (a) F. Portela-Cubillo, J. S. Scott and J. C. Walton, Chem. Commun., 2008, 2935; (b) F. Portela-Cubillo, J. S. Scott and J. C. Walton, J. Org. Chem., 2009, 74, 4934; (c) Y. P. Patil, P. J. Tambade, K. D. Parghi, R. V. Jayaram and B. M. Bhanage, Catal. Lett., 2009, 133, 201; (d) T. Mizuno, M. Mihara, T. Nakai, T. Iwai and T. Ito, Synthesis, 2007, 2524; (e) R. K. Saunthwal, M. Patel, A. K. Danodia and A. K. Verma, Org. Biomol. Chem., 2015, 13, 1521.

9 (a) Y. Lv, Y. Li, T. Xiong, W. Pu, H. Zhang, K. Sun, Q. Liu and Q. Zhang, Chem. Commun., 2013, 49, 6439; (b) C. Huang, Y. Fu, H. Fu, Y. Jiang and Y. Zhao, Chem. Commun., 2008, 6333; (c) R. Rohlmann, T. Stopka, H. Richter and O. G. Mancheño, J. Org. Chem., 2013, 78, 6050.

10 (a) C. Wang, S. Li, H. Liu, Y. Jiang and H. Fu, J. Org. Chem., 2010, 75, 7936; (b) J. Zhang, D. Zhu, C. Yu, C. Wan and Z. Wang, Org. Lett., 2010, 12, 2841; (c) L. Jiarong, C. Xian, S. Daxin, M. Shuling, L. Qing, Z. Qi and T. Jianhong, Org. Lett., 2009, 11, 1193; (d) Z. G. Li, H. Huang, H. B. Sun, H. L. Jiang and H. Liu, J. Comb. Chem., 2008, 10, 484; (e) C. C. Malakar, A. Baskakova, J. Conrad and U. Beifuss, Chem.-Eur. J., 2012, 18, 8882.

11 (a) J. Fang, J. Zhou and Z. Fang, RSC Adv., 2013, 3, 334; (b) H. Yuan, W.-J. Yoo, H. Miyamura and S. Kobayashi, Adv. Synth. Catal., 2012, 354, 2899.

12 (a) B. Han, X.-L. Yang, C. Wang, Y.-W. Bai, T.-C. Pan, X. Chen and W. Yu, J. Org. Chem., 2012, 77, 1136; (b) C. U. Maheshwari, G. S. Kumar, M. Venkateshwar, R. A. Kumar, M. L. Kantam and K. R. Reddy, Adv. Synth. Catal., 2010, 352, 341.

13 (a) A. Chanda and V. V. Fokin, Chem. Rev., 2009, 109, 725; (b) S. Chitra, N. Paul, S. Muthusbramanian and P. Manisankar, Green Chem., 2011, 13, 2777; (c) C.-J. Li, Chem. Rev., 2005, 105, 3095; (d) U. M. Lindström, Chem. Rev., 2002, 102, 2751; (e) B. Das, P. Jangili, J. Kashanna and R. A. Kumar, Synthesis, 2011, 3267. 
14 R. Breslow, Acc. Chem. Res., 1991, 24, 159.

15 (a) N. R. Modugu and P. Kumar Pittala, New J. Chem., 2017, 41, 14062; (b) H. Zeng, Q. Tian and H. Shao, Green Chem. Lett. Rev., 2011, 4, 281; (c) R. Kumar, P. Chaudhary, S. Nimesh and R. Chandra, Green Chem., 2006, 8, 356; (d) S. Chandrasekhar, C. Narsihmulu, S. S. Sultana and N. R. K. Reddy, Org. Lett., 2002, 4, 4399; (e) R. Ballini, L. Barboni and A. Palmieri, Green Chem., 2008, 10, 1004; $(f)$ A. Kamal, D. R. Reddy and S. Rajendar, Tetrahedron Lett., 2005, 46, 7951; $(g)$ S. Chandrasekhar, C. Narsihmulu, G. Chandrashekar and T. Shyamsunder, Tetrahedron Lett., 2004, 45, 2421.

16 (a) L. C. C. Vieira, M. W. Paixão and A. G. Corrêa, Tetrahedron Lett., 2012, 53, 2715; (b) K. Knop, R. Hoogenboom, D. Fisher and U. S. Schubert, Angew. Chem., Int. Ed., 2010, 49, 6288.
17 (a) N. Sharma, T. S. Chundawat, S. C. Mohapatra and S. Bhagat, RSC Adv., 2013, 3, 16336; (b) P. Kumari, N. Sharma, A. Kumar and S. C. Mahopatra, Synlett, 2017.

18 G. Karlstrom and O. Engkvist, in Theory of Poly(ethylene glycol) in Solution, American Chemical Society, 1997, vol. 680, pp. 16-30.

19 O. M. Singh, H. Junjappa and H. Ila, J. Chem. Soc., Perkin Trans. 1, 1997, 1, 3561.

20 A. F. Abdel-Magid, K. G. Carson, B. D. Harris, C. A. Maryanoff and R. D. Shah, J. Org. Chem., 1996, 61, 3849.

21 R. H. Bahekar, M. R. Jain, A. Goel, D. N. Patel, V. M. Prajapati, A. A. Gupta, P. A. Jadav and P. R. Patel, Bioorg. Med. Chem., 2007, 15, 3248. 